\title{
Global smooth solutions of Euler equations for Van der Waals gases
}

\author{
Magali Lécureux-Mercier*
}

February 17, 2011

\begin{abstract}
We prove global in time existence of solutions of the Euler compressible equations for a Van der Waals gas when the density is small enough in $\mathbf{H}^{m}$, for $m$ large enough. To do so, we introduce a specific symmetrization allowing areas of null density. Next, we make estimates in $\mathbf{H}^{m}$, using for some terms the estimates done by Grassin, who proved the same theorem in the easier case of a perfect polytropic gas. We treat the remaining terms separately, due to their nonlinearity.
\end{abstract}

2000 Mathematics Subject Classification: 35L60, 35Q31, 76N10.

Keywords: Euler compressible equations, smooth solutions, special symmetrization.

\section{Introduction}

We are interested in the Cauchy problem for Euler compressible equations, describing the evolution of a gas whose thermodynamical and kinetic properties are known at time $t=0$.

More specifically, we are concerned with the life span of smooth solutions. Various authors, in particular Sideris [20, 21], Makino, Ukai and Kawashima [13] and Chemin [2, 3] have given criteria for mathematical explosion. We know also that there exist global in time solutions for well-chosen initial data. Li [12], Serre [19] and Grassin [7] prove, for example, the global in time existence of regular solutions under some hypotheses of "expansivity".

Most of these results were obtained within the framework of perfect polytropic gases. A natural question is to determine whether these results extend to more realistic gases, following, for example, the Van der Waals law. This law takes into account the volume of molecules, which is important in physical situations like explosions or implosions. In such limits, the gas is highly compressed, and the Van der Waals law fits better with the behavior of real gases than with that of the perfect polytropic gases in such limits. The Van der Waals law is also used to modelize dusty gases, seen as perfect gases with dust pollution [8, 16, 22, 24]. This law is given by the relationship

$$
p(v-b)=\mathfrak{R} T,
$$

where $p$ is the pressure, $v$ is the massic volume, $T$ is the temperature, and $b, \Re$ are given constants.

The addition of the covolume $b$, representing the compressibility limit of the molecules in the gas, modifies nontrivially the analysis of the Euler equations. However, we are going to show the global in time existence of regular solutions, thus generalizing a theorem of Grassin [7].

Theorem 1.1. Let $m>1+d / 2$. Let $\left(\rho_{0}, u_{0}, s_{0}\right)$ be the initial conditions for the Cauchy problem associated with the Euler compressible equations (2.1) for a Van der Waals gas with constant $c_{v}$ (see (2.6) below) and $c_{v}>0$. Let us assume $0 \leqslant \rho_{0} \leqslant 1 / b$. Then we can define $\gamma_{0}=1+\frac{\mathfrak{R}}{c_{v}}$; furthermore there exists $\varepsilon_{0}>0$ such that if

\footnotetext{
*Laboratoire MAPMO, Université d'Orléans, Rue de Chartres B.P. 6759, 45067 Orléans cedex 2, France, E-mail: magali.lecureux-mercier@univ-orleans.fr
} 
(H1) $\left\|\left(\pi_{0}, s_{0}\right)\right\|_{\mathbf{H}^{m}\left(\mathbb{R}^{d}\right)} \leqslant \varepsilon_{0}$, where $\pi_{0}=\left(\frac{\rho_{0}}{1-b \rho_{0}}\right)^{\frac{\gamma_{0}-1}{2}} \exp \left(\frac{s_{0}}{c_{v}}\right)$,

(H2) the initial speed $u_{0}$ belongs to the space $X=\left\{z: \mathbb{R}^{d} \rightarrow \mathbb{R}^{d} ; \mathrm{D} z \in \mathbf{L}^{\infty}, \mathrm{D}^{2} z \in \mathbf{H}^{m-1}\right\}$,

(H3) there exists $\delta>0$ such that for all $x \in \mathbb{R}^{d}$, $\operatorname{dist}\left(\operatorname{Spec}\left(\mathrm{D} u_{0}\right)(x), \mathbb{R}^{-}\right) \geqslant \delta$, where $\operatorname{Spec}(M)$ stands for the spectrum of the matrix $M \in \mathcal{M}_{d}(\mathbb{R})$,

(H4) the initial density $\rho_{0}$ and the initial entropy $s_{0}$ have compact support,

then the problem

$$
\left\{\begin{array}{cccc}
\partial_{t} \bar{u}+(\bar{u} \cdot \nabla) \bar{u} & = & 0 & \text { on } \mathbb{R}^{+} \times \mathbb{R}^{d}, \\
\bar{u}(0, x) & = & u_{0}(x) & \text { on } \mathbb{R}^{d} .
\end{array}\right.
$$

admits a global classical solution. If, furthermore, $\gamma_{0}=\frac{\nu+1}{\nu-1}$ with $\nu \in \mathbb{N}$ and $\nu \geqslant 2$, or if $\gamma_{0}$ and $m$ satisfy $\nu=\frac{\gamma_{0}+1}{\gamma_{0}-1} \geqslant m>1+\frac{d}{2}$, then there exists a global classical solution $(\rho, u, s)$ to the Euler compressible equations (2.1) satisfying

$$
\left(\left(\frac{\rho}{1-b \rho}\right)^{\frac{\gamma_{0}-1}{2}}, u-\bar{u}, s\right) \in \mathscr{C}^{0}\left(\mathbb{R}^{+} ; \mathbf{H}^{m}\left(\mathbb{R}^{d} ; \mathbb{R}^{d+2}\right)\right) \cap \mathscr{C}^{1}\left(\mathbb{R}^{+} ; \mathbf{H}^{m-1}\left(\mathbb{R}^{d} ; \mathbb{R}^{d+2}\right)\right) .
$$

To do so, we have first to extend to Van der Waals gases a symmetrization obtained by Makino, Ukai and Kawashima [13, which allows null density areas. Next we will derive energy estimates in $\mathbf{H}^{m}$.

Since the Van der Waals gases have a behavior close to that of perfect polytropic gases for weak densities, this result is not very surprising. However, the nonlinear terms introduced by the Van der Waals law have to be treated carefully.

In section 2, we describe the thermodynamic properties of a compressible gas, and we state some important properties such as the Friedrichs symmetrization. In section 3, we give the detailed proof of this result, and in ection 4 we give the proofs of some technical lemmas used in section 3 ,

\section{Thermodynamic and first properties}

\subsection{Conservation law}

Compressible fluid dynamics, without viscosity or heat transfer, is described by the Euler equations, which are made of the conservation of mass, momentum, and energy (see [5, Chap. 2]):

$$
\left\{\begin{array}{l}
\partial_{t} \rho+\operatorname{div}(\rho u)=0 \\
\partial_{t} q+\operatorname{div}(\rho u \otimes u)+\nabla p=0 \\
\partial_{t} E+\operatorname{div}((E+p) u)=0
\end{array}\right.
$$

where $\rho$ is the mass of the fluid per unit of volume, $q=\rho u$ is the momentum per unit of volume, and $E=\frac{1}{2} \rho u^{2}+\rho e$ is the total energy per unit of volume, sum of the kinetic energy and internal energy. This is a system of $(d+2)$ equations and $(d+3)$ unknowns: the density $\rho \in \mathbb{R}^{+}$, the speed $u \in \mathbb{R}^{d}$, the internal energy $e \in \mathbb{R}$, and the pressure $p \in \mathbb{R}$. In order to complete this system, we have to add a state law, for example, an incomplete state law, also called pressure law $(\rho, e) \mapsto p(\rho, e)$.

Definition 2.1. We denote as Euler compressible equations the system composed of the conservation laws (2.1) and an incomplete state law $p=p(\rho, e)$.

A simplified model is often considered, conserving only the conservation of mass and momentum, assuming that the fluid is isentropic. This simplified system is

$$
\left\{\begin{array}{l}
\partial_{t} \rho+\operatorname{div}(\rho u)=0 \\
\partial_{t} q+\operatorname{div}(\rho u \otimes u)+\nabla p=0
\end{array}\right.
$$


and the state law is a given function $\rho \mapsto p(\rho)$.

Definition 2.2. We denote as isentropic Euler equations the system (2.2) with a given state law $p=p(\rho)$ such that

$$
p=-\left.\frac{\partial e}{\partial v}\right|_{s},
$$

where $v=1 / \rho$ is the specific volume, $T$ is the temperature, $s$ is the specific entropy, and $f=e-T s$ is the specific free energy, assuming we are given a complete equation of state $(v, s) \mapsto e(v, s)$.

The thermodynamical variables $v, s, e, T, p$ must satisfy some relations described in section 2.2 The Euler equations are a system of first order conservation law, whose study is developed in particular in the books [4, 17, 18].

\section{$2.2 \quad$ State law}

The state law has a strong influence on the mathematical analysis of the compressible Euler equations. The state law of "real" gases can reveal particular behavior and introduce existence and/or uniqueness troubles which do not appear for perfect gases; see [14]. We describe below the physical principles a state law has to satisfy.

\subsubsection{Definitions}

We consider a fluid, whose internal energy is a regular function of its specific volume $1 v=1 / \rho$ and its specific entropy $s$. This means that the gas is endowed with a complete state law, or energy law $e=e(v, s)$. The fundamental thermodynamic principle is then

$$
\mathrm{d} e=-p \mathrm{~d} v+T \mathrm{~d} s,
$$

where $p$ is the pressure and $T$ the temperature of the gas. Consequently, the pressure $p$ and the temperature $T$ can be defined as

$$
p=-\left.\frac{\partial e}{\partial v}\right|_{s}, \quad T=\left.\frac{\partial e}{\partial s}\right|_{v},
$$

where the notation $\mid$ precises the variable maintained constant in the partial derivation.

The greater order derivatives of $e$ also have an important role; we introduce the following adimensional quantities:

$$
\gamma=-\left.\frac{v}{p} \frac{\partial p}{\partial v}\right|_{s}, \quad \Gamma=-\left.\frac{v}{T} \frac{\partial T}{\partial v}\right|_{s}, \quad \delta=\left.\frac{p v}{T^{2}} \frac{\partial T}{\partial s}\right|_{v}, \quad \mathscr{G}=-\left.\frac{v}{2} \frac{\left.\frac{\partial^{3} e}{\partial v^{3}}\right|_{s}}{\frac{\partial^{2} e}{\partial v^{2}}}\right|_{s} .
$$

The coefficient $\gamma$ is called the adiabatic exponent, and $\Gamma$ is the Grüneisen coefficient. The quantities $\gamma, \delta, \Gamma$, and $\mathscr{G}$ characterize the geometrical properties of the isentropic curves in the $(v, p)$ plane (see [14]). They are related to $e$ through the relationships

$$
\gamma=\frac{v}{p} \frac{\partial^{2} e}{\partial v^{2}}, \quad \quad \quad \Gamma=-\frac{v}{T} \frac{\partial^{2} e}{\partial s \partial v}, \quad \delta=\frac{p v}{T^{2}} \frac{\partial^{2} e}{\partial s^{2}} .
$$

We also introduce the calorific capacity at constant volume $c_{v}$ and the calorific capacity at constant pressure $c_{p}$ by

$$
c_{v}=\left.\frac{\partial e}{\partial T}\right|_{v}=\frac{T}{\left.\frac{\partial^{2} e}{\partial s^{2}}\right|_{v},} \quad c_{p}=\left.T \frac{\partial s}{\partial T}\right|_{p} .
$$

\footnotetext{
${ }^{1}$ specific is a synonym of massic.
} 
These two quantities are linked with $\frac{p v}{T}$ and with $\gamma, \delta, \Gamma$ through

$$
\delta c_{v}=\frac{p v}{T}, \quad c_{p}=\frac{p v}{T} \frac{\gamma}{\gamma \delta-\Gamma^{2}} .
$$

The quantity $\gamma_{*}=\frac{c_{p}}{c_{v}}$ can also be expressed as $\gamma_{*}=\frac{\gamma \delta}{\gamma \delta-\Gamma^{2}}$. It is not equal to $\gamma$ in the general case, but for an ideal gas we have $\delta=\Gamma=\gamma-1$, so that $\gamma_{*}=\gamma$.

\subsubsection{Thermodynamical constraints}

It is very natural to assume $v \geqslant 0$. We assume furthermore that the pressure $p$ and the temperature $T$ are positive, which imposes that $e$ is a function increasing in $T$ and decreasing in $v$.

A classical thermodynamical hypothesis additionaly requires $e$ to be a convex function of $s$ and $v$, which means that

$$
\gamma \delta-\Gamma^{2} \geqslant 0, \quad \delta \geqslant 0, \quad \gamma \geqslant 0 .
$$

In particular, $\gamma \geqslant 0$ means that $p$ increases with the density $\rho=1 / v$, which allows us to define the adiabatic sound speed by

$$
c=\sqrt{\left.\frac{\partial p}{\partial \rho}\right|_{s}}=\sqrt{\gamma \frac{p}{\rho}} .
$$

Then, $\mathscr{G}$ can be rewritten using $\rho$ and $c$ as $\mathscr{G}=\left.\frac{1}{c} \frac{\partial(\rho c)}{\partial \rho}\right|_{s}$.

Furthermore, we usually require $\Gamma>0$ and $\mathscr{G}>0$. The condition $\Gamma>0$ is not thermodynamically required but is satisfied for many gases and ensures that the isentropes do not cross each other in the $(v, p)$ plane. The condition $\mathscr{G}>0$ means that the isentropes are strictly convex in the $(v, p)$ plane.

\subsubsection{Van der Waals gas}

Definition 2.3. A gas is said to follow the Van der Waals law if it satisfies the following pressure law:

$$
p(v-b)=\mathfrak{R} T,
$$

where $v$ is the massic volume and $b$ is the covolume, representing the compressibility limit of the fluid, due to the volume of the molecules.

The Van der Waals law is a modification of the perfect gas law, in which $b=0$. In opposition to the perfect gas law, it takes into account the proper size of the molecules, which is important in some situations when the gas is strongly compressed. In this model, the density must be bounded, and the maximal density is $\rho_{\max }=\frac{1}{b}$.

The fundamental relationship (2.3) gives us the PDE $\partial_{v} e+\frac{\Re}{v-b} \partial_{s} e=0$. Thus, we introduce new variables $w=(v-b)^{-\mathfrak{R}}, \sigma=(v-b)^{-\mathfrak{R}} \exp (s)$, and $\hat{e}(w, \sigma)=e(v, s)$. We obtain $\partial_{w} \hat{e}=0$, so that $e=\mathcal{E}\left((v-b)^{-\mathfrak{R}} \exp (s)\right)$ for any regular function $\mathcal{E}$.

If we assume furthermore that $c_{v}$ is constant, thanks to the definition of $c_{v}$ and (2.3), we get that $\left.\frac{\partial^{2} e}{\partial s^{2}}\right|_{v}=\left.\frac{1}{c_{v}} \frac{\partial e}{\partial s}\right|_{v} ;$ hence $\sigma \mathcal{E}^{\prime \prime}=\left(\frac{1}{c_{v}}-1\right) \mathcal{E}^{\prime}$ and $\mathcal{E}(\sigma)=C \sigma^{1 / c_{v}}$, which leads to

$$
e=(v-b)^{-\frac{\Re}{c_{v}}} \exp \left(\frac{s}{c_{v}}\right), \quad \quad p=\frac{\Re}{c_{v}} \frac{e}{v-b} .
$$

Some computations allow us to finally obtain

$$
\gamma=\gamma_{0} \frac{v}{v-b}, \quad \quad \Gamma=\delta=\left(\gamma_{0}-1\right) \frac{v}{v-b}, \quad \mathscr{G}=\frac{\gamma_{0}+1}{2} \frac{v}{v-b},
$$


where

$$
\gamma_{0}:=\frac{\Re}{c_{v}}+1
$$

The conditions of section 2.2.2 are then satisfied for $\gamma_{0}>1$.

\section{Remark.}

1. A perfect gas can be seen as a Van der Waals gas with $b=0$. A perfect gas for which $c_{v}$ is constant is called polytropic.

2. The Van der Waals law coincides with the dusty gas law [8, 9, 16, 22, 24]. In this model, we consider that the gas is perfect but polluted by dust particles that are equidistributed and have a nonnegligible volume.

Very often in the literature, the perfect polytropic gases are considered as a canonical example. However, their adequation with physical observations is not as good as for Van der Waals gases, for example, in explosion phenomena or in the sonoluminescence phenomenon [1, 6, 11.

In the following, we consider only Van der Waals fluids with constant and strictly positive calorific capacity $c_{v}$ :

$$
c_{v}>0,
$$

which implies $\gamma_{0}>1$.

\subsection{Symmetrization}

An important property of the Euler equations is their symmetrizability.

\subsubsection{General case, without vacuum}

If $\rho>0$ and $\left.\frac{\partial p}{\partial \rho}\right|_{s}>0$, then the system (2.1) can be written in the variables $(p, \rho, s)$. Then, the system is almost symmetric, since it can be written matricially as $\partial_{t} \tilde{V}+\sum_{k} \tilde{A}_{k}(\tilde{V}) \partial_{k} \tilde{V}=0$, with $\tilde{V}=\left(p, u^{\mathbf{T}}, s\right)^{\mathbf{T}}$ and

$$
\tilde{A}(\xi, V)=\sum_{k} \xi_{k} \tilde{A}_{k}(\tilde{V})=\left(\begin{array}{ccc}
u \cdot \xi & \rho c^{2} \xi^{\mathbf{T}} & 0 \\
\frac{1}{\rho} \xi & u \cdot \xi I_{d} & 0 \\
0 & 0 & u \cdot \xi
\end{array}\right)
$$

This matrix is almost symmetric since we obtain a symmetric matrix by multiplying it on the left by $D:=\operatorname{diag}\left(\frac{1}{\rho c^{2}}, \rho, \ldots, \rho, 1\right)$. Consequently, we have the following proposition.

Proposition 2.4. The system (2.1) is Friedrichs symmetrizable when $(\rho, u, s)$ takes values in a compact subset of $\mathbb{R}_{+}^{*} \times \mathbb{R}^{d} \times \mathbb{R}$.

Indeed, for such values of $(\rho, u, s), D \tilde{A}(\xi, V)$ is symmetric with $D$ symmetric positive-definite.

\subsubsection{Van der Waals gas}

We are not completely satisfied with the previous formulation as it does not authorize $\rho$ to vanish or even to tend to 0 at infinity. For example, $\rho$ cannot be taken into $\mathbf{H}^{m}$ for $m \geqslant 0$. Makino, Ukai and Kawashima have introduced in [13] a symmetrization for perfect polytropic gases allowing the null density areas. Here we generalize their method to the case of Van der Waals gases. 
Let us recall first that, for Van der Waals gases, $\gamma=\frac{\gamma_{0}}{1-b \rho}$ and $p=\left(\gamma_{0}-1\right)\left(\frac{\rho}{1-b \rho}\right)^{\gamma_{0}} \exp \left(\frac{s}{c_{v}}\right)$. We now introduce the new variable

$$
\pi=2 \sqrt{\frac{\gamma_{0}}{\gamma_{0}-1}}\left(\frac{p}{\gamma_{0}-1}\right)^{\frac{\gamma_{0}-1}{2 \gamma_{0}}},
$$

and we rewrite the system (2.1) in the variables $(\pi, u, s)$. In order to do that, we first write the system (2.1) in $(p, u, s)$ variables:

$$
\left\{\begin{array}{l}
\partial_{t} p+u \cdot \nabla p+\rho c^{2} \operatorname{div} u=0 \\
\partial_{t} u+(u \cdot \nabla) u+\frac{1}{\rho} \nabla p=0 \\
\partial_{t} s+u \cdot \nabla s=0
\end{array}\right.
$$

Since $\pi=f(p)=C p^{\alpha}$, it is sufficient to multiply the first line by $f^{\prime}(p)=C \alpha p^{\alpha-1}$ to obtain an equation in $\pi$ :

$$
\left\{\begin{aligned}
\partial_{t} \pi+u \cdot \nabla \pi+C \alpha p^{\alpha-1} \rho c^{2} \operatorname{div} u & =0 \\
\partial_{t} u+(u \cdot \nabla) u+\frac{1}{\rho C \alpha p^{\alpha-1}} \nabla \pi & =0 \\
\partial_{t} s+u \cdot \nabla s & =0
\end{aligned}\right.
$$

Besides, we know that $c^{2}=\frac{\gamma_{0}}{1-b \rho} \frac{p}{\rho}, C=2 \sqrt{\frac{\gamma_{0}}{\gamma_{0}-1}}\left(\frac{1}{\gamma_{0}-1}\right)^{\frac{\gamma_{0}-1}{2 \gamma_{0}}}$, and $\alpha=\frac{\gamma_{0}-1}{2 \gamma_{0}}$. It remains to evaluate the coefficients:

$$
\begin{aligned}
C \alpha p^{\alpha-1} \rho c^{2} & =\frac{\gamma_{0} \alpha}{1-b \rho} \pi \\
& =\frac{\gamma_{0}-1}{2} \frac{\pi}{1-b \rho}, \\
\frac{1}{\rho C \alpha p^{\alpha-1}} & =\frac{\exp \left(s /\left(\gamma_{0} c_{v}\right)\right)}{1-b \rho} \frac{\left(\gamma_{0}-1\right)^{1 / \gamma_{0}}}{\alpha C^{\left(\gamma_{0}-1\right) /\left(\gamma_{0} \alpha\right)}} \pi^{\frac{1-\alpha-1 / \gamma_{0}}{\alpha}} \\
& =\exp \left(\frac{s}{\gamma_{0} c_{v}}\right) \frac{\gamma_{0}-1}{2} \frac{\pi}{1-b \rho} .
\end{aligned}
$$

Thus,

$$
\left\{\begin{aligned}
\partial_{t} \pi+u \cdot \nabla \pi+\frac{\gamma_{0}-1}{2} \frac{\pi}{1-b \rho} \operatorname{div} u & =0 \\
\partial_{t} u+(u \cdot \nabla) u+\frac{\gamma_{0}-1}{2} \frac{\pi}{1-b \rho} \exp \left(\frac{s}{\gamma_{0} c_{v}}\right) \nabla \pi & =0 \\
\partial_{t} s+u \cdot \nabla s & =0 .
\end{aligned}\right.
$$

Moreover, $1 /(1-b \rho)=1+b\left(\frac{\gamma_{0}-1}{4 \gamma_{0}}\right)^{\frac{1}{\gamma_{0}-1}} \exp \left(\frac{-s}{\gamma_{0} c_{v}}\right) \pi^{\frac{2}{\gamma_{0}-1}}$. Therefore, denoting

$$
\nu=\frac{\gamma_{0}+1}{\gamma_{0}-1}>1 \quad \text { and } \quad \tilde{b}=b\left(\frac{\gamma_{0}-1}{4 \gamma_{0}}\right)^{\frac{1}{\gamma_{0}-1}},
$$

we obtain $\frac{\pi}{1-b \rho}=\pi\left(1+\tilde{b} e^{-s /\left(\gamma_{0} c_{v}\right)} \pi^{\nu-1}\right)$, which is well defined for all $\pi \geqslant 0$ and in particular for $p=0$, since we have assumed in (2.11) that $\gamma_{0}>1$. The matrix associated with the system (2.13) is now written as

$$
A(\xi, \pi, u, s)=\left(\begin{array}{ccc}
u \cdot \xi & \frac{\gamma_{0}-1}{2} \frac{\pi}{1-b \rho} \xi^{\mathbf{T}} & 0 \\
\frac{\gamma_{0}-1}{2} \frac{\pi}{1-b \rho} \exp \left(\frac{s}{\gamma_{0} c_{v}}\right) \xi & u \cdot \xi I_{d} & 0 \\
0 & 0 & u \cdot \xi
\end{array}\right) .
$$

It is once again "almost symmetric" in the sense that $S A$ is symmetric, $S$ being the diagonal definite positive matrix

$$
S=\operatorname{diag}\left(1, \exp \left(-s /\left(\gamma_{0} c_{v}\right)\right), \ldots, \exp \left(-s /\left(\gamma_{0} c_{v}\right)\right), 1\right) .
$$

Furthermore, this symmetrizer is independent of $p$ and in particular is well defined and definite positive even when $p$ or $\rho$ vanishes. Finally, we have the following proposition. 
Proposition 2.5. For a Van der Waals gas with constant $c_{v}$ and $c_{v}>0$, the system of Euler equations can be written for regular solutions as (2.13), which is Friedrichs symmetrizable for $(p, u, s) \in \mathcal{K}$, where $\mathcal{K}$ is a compact subset of $\mathbb{R}^{+} \times \mathbb{R}^{d} \times \mathbb{R}$.

Note that in the variables $(\pi, u, s)$, the system (2.13) can be written as

$$
\left\{\begin{aligned}
\partial_{t} \pi+u \cdot \nabla \pi+\frac{\gamma_{0}-1}{2}\left(1+\tilde{b} \exp \left(\frac{-s}{\gamma_{0} c_{v}}\right) \pi^{\nu-1}\right) \pi \operatorname{div} u & =0, \\
\partial_{t} u+(u \cdot \nabla) u+\frac{\gamma_{0}-1}{2} \exp \left(\frac{s}{\gamma_{0} c_{v}}\right)\left(1+\tilde{b} \exp \left(\frac{-s}{\gamma_{0} c_{v}}\right) \pi^{\nu-1}\right) \pi \nabla \pi & =0, \\
\partial_{t} s+u \cdot \nabla s & =0,
\end{aligned}\right.
$$

where $\nu$ and $\tilde{b}$ are defined as in (2.14).

\subsection{Local existence}

The symmetrization of Proposition 2.5 is very useful in showing local existence of regular solutions with vanishing density.

Theorem 2.6. We consider a Van der Waals gas with constant $c_{v}$ such that $\left.\left.\gamma_{0} \in\right] 1,3\right]$. Let

$$
\pi_{0}=2 \sqrt{\frac{\gamma_{0}}{\gamma_{0}-1}}\left(\frac{\rho_{0}}{1-b \rho_{0}}\right)^{\left(\gamma_{0}-1\right) / 2} \exp \left(\frac{\gamma_{0}-1}{2 \gamma_{0} c_{v}} s_{0}\right)
$$

where $\rho_{0} \in \mathscr{C}^{1}\left(\mathbb{R}^{d} ;\left[0,1 / b[)\right.\right.$. We also introduce $\nu=\frac{\gamma_{0}+1}{\gamma_{0}-1}, \tilde{b}=b\left(\frac{\gamma_{0}-1}{4 \gamma_{0}}\right)^{\frac{1}{\gamma_{0}-1}}$. We assume that $\left(\pi_{0}, u_{0}, s_{0}\right) \in \mathbf{H}^{m}\left(\mathbb{R}^{d}\right)$ for $m>1+\frac{d}{2}$. Then there exist $T>0$ and a unique solution $(\pi, u, s) \in$ $\mathscr{C}^{1}\left([0, T] \times \mathbb{R}^{d}\right)$ to the Cauchy problem for

$$
\left\{\begin{aligned}
\partial_{t} \pi+u \cdot \nabla \pi+\frac{\gamma_{0}-1}{2}\left(1+\tilde{b} \exp \left(\frac{-s}{\gamma_{0} c_{v}}\right) \pi^{\nu-1}\right) \pi \operatorname{div} u & =0, \\
\partial_{t} u+(u \cdot \nabla) u+\frac{\gamma_{0}-1}{2} \exp \left(\frac{s}{\gamma_{0} c_{v}}\right)\left(1+\tilde{b} \exp \left(\frac{-s}{\gamma_{0} c_{v}}\right) \pi^{\nu-1}\right) \pi \nabla \pi & =0, \\
\partial_{t} s+u \cdot \nabla s & =0,
\end{aligned}\right.
$$

with initial condition $\left(\pi_{0}, u_{0}, s_{0}\right)$. Furthermore,

$$
(\pi, u, s) \in \mathscr{C}\left([0, T] ; \mathbf{H}^{m}\left(\mathbb{R}^{d} ; \mathbb{R}^{d+2}\right)\right) \cap \mathscr{C}^{1}\left([0, T] ; \mathbf{H}^{m-1}\left(\mathbb{R}^{d} ; \mathbb{R}^{d+2}\right)\right) .
$$

\subsection{Positivity of the density}

For regular solutions, the positivity of the density is given by the following

Proposition 2.7. Let $(\bar{\rho}, \bar{u}, \bar{s}) \in \mathscr{C}^{1}\left(\left[0, T_{\mathrm{ex}}\left[\times \mathbb{R}^{d}\right)\right.\right.$ be a regular solution of the Cauchy problem for (2.1) associated with the regular initial conditions $\left(\rho_{0}, u_{0}, s_{0}\right) \in \mathscr{C}^{1}\left(\mathbb{R}^{d}\right)$. If $\nabla \bar{u} \in \mathbf{L}^{\infty}\left([0, T] \times \mathbb{R}^{d}\right)$ for all $T<T_{\mathrm{ex}}, \rho_{0} \in \mathbf{L}^{\infty}\left(\mathbb{R}^{d}, \mathbb{R}\right)$, and if $\rho_{0}(x) \geqslant 0$ for all $x \in \mathbb{R}^{d}$, then for all $t \in\left[0, T_{\mathrm{ex}}[\right.$,

$$
\rho(t, x) \geqslant 0 \text {. }
$$

Proof. We use the method of characteristics to obtain an expression of the solution of the Cauchy problem for (1.1). Assuming that $\nabla \bar{u}$ is bounded, we obtain

$$
\rho(t, x)=\rho_{0}(X(0 ; t, x)) \exp \left(-\int_{0}^{t} \operatorname{div} \bar{u}(\tau, X(\tau ; t, x)) \mathrm{d} \tau\right)>0,
$$

where $X$ is a solution of the Cauchy problem

$$
\frac{\mathrm{d} X}{\mathrm{~d} t}=\bar{u}(t, X), \quad X\left(t_{0} ; t_{0}, x_{0}\right)=x_{0},
$$

which is global in time since $\nabla \bar{u}$ is bounded. 
We also prove that for a Van der Waals gas with constant $c_{v}>0$, the variable $\pi$ introduced in section 2.3.2 defined by

$$
\pi=2 \sqrt{\frac{\gamma_{0}}{\gamma_{0}-1}}\left(\frac{p}{\gamma_{0}-1}\right)^{\frac{\gamma_{0}-1}{2 \gamma_{0}}}
$$

remains nonnegative if it is nonnegative at initial time. This property implies in particular that if $\rho_{0}<1 / b$, then, as long as the regular solution exists, this property will be satisfied.

Proposition 2.8. We consider a Van der Waals gas with constant $c_{v}$. We denote $\pi=2 \sqrt{\frac{\gamma_{0}}{\gamma_{0}-1}}\left(\frac{p}{\gamma_{0}-1}\right)^{\frac{\gamma_{0}-1}{2 \gamma_{0}}}$, $\nu=\frac{\gamma_{0}+1}{\gamma_{0}-1}$, and $\tilde{b}=b\left(\frac{\gamma_{0}-1}{4 \gamma_{0}}\right)^{\frac{1}{\gamma_{0}-1}}$. Let $(\bar{\pi}, \bar{u}, \bar{s}) \in \mathscr{C}^{1}\left(\left[0, T_{\mathrm{ex}}\left[\times \mathbb{R}^{d}\right)\right.\right.$ be a regular solution of (2.15) satisfying the initial conditions

$$
\pi(0, x)=\pi_{0}(x), \quad u(0, x)=u_{0}(x), \quad s(0, x)=s_{0}(x),
$$

with $\left(\pi_{0}, u_{0}, s_{0}\right) \in \mathscr{C}^{1}\left(\mathbb{R}^{d}\right)$ and $s_{0} \in \mathbf{L}^{\infty}$. If $\operatorname{div} \bar{u} \in \mathbf{L}^{\infty}\left([0, T] \times \mathbb{R}^{d}\right)$ for all $T<T_{\mathrm{ex}}, \pi_{0} \in \mathbf{L}^{\infty}\left(\mathbb{R}^{d}, \mathbb{R}\right)$ and $0 \leqslant \rho_{0}(x)<1 / b$ for all $x \in \mathbb{R}^{d}$, then for all $t \in\left[0, T_{\mathrm{ex}}[, \pi \geqslant 0\right.$. Then, we can define $\rho$, and we have

$$
0 \leqslant \rho(t, x)<1 / b .
$$

Proof. Let $T_{0}<T_{\mathrm{ex}}$. We introduce the Cauchy problem

$$
\begin{aligned}
\partial_{t} w+\operatorname{div}(w \bar{u}) & =g(t, x, w), \\
w(0, x) & =w_{0}(x),
\end{aligned}
$$

where

$$
g(t, x, w)=\left(1-\frac{\gamma_{0}-1}{2}\left(1+\tilde{b} \exp \left(\frac{-\bar{s}(t, x)}{\gamma_{0} c_{v}}\right) w^{\nu-1}\right)\right) w \operatorname{div} \bar{u}(t, x) .
$$

We can apply the Kružkov theorem [10, 17]. Indeed, the hypotheses ensure that $g(t, x, w)-$ $w \operatorname{div}(\bar{u}(t, x))=-\frac{\gamma_{0}-1}{2}\left(1+\tilde{b} e^{-\bar{s} /\left(\gamma_{0} c_{v}\right)} w^{\nu-1}\right) w \operatorname{div} \bar{u}$ is uniformly bounded with respect to $x \in \mathbb{R}^{d}$ when $w$ is considered as a variable taking values in a compact set. Furthermore, $\pi_{0} \in \mathbf{L}^{\infty}$, so the regular solution $\bar{\pi}$ coincides with the entropy solution $w_{1}$ of (2.17) associated with $w_{0,1}=\pi_{0}$.

In addition, the entropy solution $w_{2}$ of (2.17) associated with the initial condition $w_{0,2} \equiv 0$ is the function constantly equal to 0 .

According to Kružkov theorem $w_{0,1} \geqslant w_{0,2}$ implies $w_{1} \geqslant w_{2}$ for all $(t, x) \in\left[0, T_{0}\right] \times \mathbb{R}^{d}$; that is, $\bar{\pi}(t, x) \geqslant 0$ for all $(t, x) \in\left[0, T_{0}\right] \times \mathbb{R}^{d}$. The formula

$$
\rho=\frac{1}{b}\left(1-\frac{1}{1+\tilde{b} \exp \left(\frac{-s}{\gamma_{0} c_{v}}\right) \pi^{\frac{2}{\gamma_{0}-1}}}\right)
$$

allows us to conclude the proof.

\section{Proof of Theorem 1.1}

In order to prove this theorem, we adapt Grassin's idea [7. First, we look to the isentropic case, which allows us to simplify the estimates. For a Van der Waals gas, nonlinear terms now appear in the estimate which we need to treat separately. 


\subsection{Isentropic case}

Let us first consider the isentropic case

$$
\left\{\begin{aligned}
\partial_{t} \pi+u \cdot \nabla \pi+\frac{\gamma_{0}-1}{2}\left(1+\tilde{b} \pi^{\nu-1}\right) \pi \operatorname{div} u & =0 \\
\partial_{t} u+(u \cdot \nabla) u+\frac{\gamma_{0}-1}{2}\left(1+\tilde{b} \pi^{\nu-1}\right) \pi \nabla \pi & =0
\end{aligned}\right.
$$

with initial conditions

$$
\pi(0, x)=\pi_{0}(x), \quad u(0, x)=u_{0}(x),
$$

which is technically simpler than the general case but provides estimates very useful in treating the general case.

We also consider the problem

$$
\left\{\begin{array}{cccc}
\partial_{t} \bar{u}+(\bar{u} \cdot \nabla) \bar{u} & = & 0 & \text { on } \mathbb{R}^{+} \times \mathbb{R}^{d}, \\
\bar{u}(0, x) & = & u_{0}(x) & \text { on } \mathbb{R}^{d}
\end{array}\right.
$$

obtained by neglecting $\pi$ in (3.1). According to [7, Lemma 3.1 and Prop. 3.1] we have the following preliminary result.

Proposition 3.1. Under hypotheses (H2) and (H3), the problem (3.3) admits a global regular solution $\bar{u}$ satisfying

1. $\mathrm{D} \bar{u}(t, x)=\frac{\mathrm{Id}}{1+t}+\frac{K(t, x)}{(1+t)^{2}}$ for all $x \in \mathbb{R}^{d}$ and $t \in \mathbb{R}^{+}$,

2. $\left\|\mathrm{D}^{l} \bar{u}(t, \cdot)\right\|_{\mathbf{L}^{2}} \leqslant K_{l}(1+t)^{d / 2-(l+1)}$ for $l \in \llbracket 2, m+1 \rrbracket$,

3. $\left\|\mathrm{D}^{2} \bar{u}(t, \cdot)\right\|_{\mathbf{L}^{\infty}} \leqslant C(1+t)^{-3}$,

where $K: \mathbb{R}^{+} \times \mathbb{R}^{d} \rightarrow \mathcal{M}_{d}(\mathbb{R}),\|K\|_{\mathbf{L}^{\infty}\left(\mathbb{R}^{+} \times \mathbb{R}^{d}\right)} \leqslant K_{1}$, and $C$ and $K_{l}$ for $l \in \llbracket 1, m+1 \rrbracket$ are nonnegative constants not depending on $m, d, \delta,\left\|u_{0}\right\|_{X}$.

\subsubsection{Local uniqueness}

Proposition 3.2. Let $U_{0}=\left(\pi_{0}, u_{0}\right)^{\mathbf{T}} \in \mathbf{H}^{m}\left(\mathbb{R}^{d}\right)$ and $\tilde{U}_{0}$ be two initial data for (3.1). Let $U=$ $(\pi, u)^{\mathbf{T}}, \tilde{U}$ be two corresponding solutions, defined for $0 \leqslant t \leqslant T_{0}$. We assume that $\|\mathrm{D} \tilde{U}\|_{\mathbf{L}_{\left(\left[0, T_{0}\right] \times \mathbb{R}^{d}\right)}<}<$ $\infty$ and that $\tilde{\pi}^{\nu-1} \in \mathbf{L}^{\infty}\left(\left[0, T_{0}\right] \times \mathbb{R}^{d}\right)$. Let $x_{0} \in \mathbb{R}^{d}$ and $R \geqslant 0$. We denote

$$
\begin{aligned}
M & =\sup \left\{\left(\frac{\gamma_{0}-1}{2}|\pi|\left(1+\tilde{b} \pi^{\nu-1}\right)+|u|\right)(t, x),(t, x) \in\left[0, T_{0}\right] \times B\left(x_{0}, R\right)\right\}, \\
C_{T} & =\left\{(t, x) \in[0, T] \times B\left(x_{0}, R-M t\right)\right\} \quad \text { for } T \in\left[0, T_{1}\right],
\end{aligned}
$$

where $T_{1}=\min \left(T_{0}, R / M\right)$. If $U_{0}=\tilde{U}_{0}$ on $B\left(x_{0}, R\right)$, then $U=\tilde{U}$ on $C_{T_{1}}$.

The proof of this proposition is classical for hyperbolic systems (see, for example, [15]), the constant $M$ being the maximal propagation speed.

Proof. Let $U_{0}=\left(\pi_{0}, u_{0}\right), \tilde{U}_{0}=\left(\tilde{\pi}_{0}, \tilde{u}_{0}\right)$ be two initial data for (3.1) such that $U_{0} \in \mathbf{H}^{m}$. Let $U, \tilde{U}$ be solutions of the associated Cauchy problems. We assume that these solutions are defined on $\left[0, T_{0}\right]$ with $T_{0}>0$. Let also $x_{0} \in \mathbb{R}^{d}, R \in \mathbb{R}_{+}^{*}$, and $M, C_{T}$ be as in (3.4) and (3.5). Note first that, as $U_{0} \in \mathbf{H}^{m}\left(\mathbb{R}^{d}\right)$, then $U \in \mathscr{C}^{0}\left(\left[0, T_{0}\right] ; \mathbf{H}^{m}\left(\mathbb{R}^{d}\right)\right)$ and, consequently, $\|\mathrm{D} U\|_{\mathbf{L}^{\infty}\left(C_{T}\right)}$ is bounded and $\pi^{\nu-1} \in \mathbf{L}^{\infty}\left(C_{T_{0}}\right)$.

We have

$$
\partial_{t} U+\sum_{j} a_{j}(U) \partial_{j} U=0
$$


where, denoting $\left(\mathbf{e}_{\mathbf{j}}\right)_{1 \leqslant j \leqslant d}$ the standard orthonormal basis of $\mathbb{R}^{d}$,

$$
a_{j}(U)=\left(\begin{array}{cc}
u_{j} & \frac{\gamma_{0}-1}{2}\left(\pi+\tilde{b} \pi^{\nu}\right) \mathbf{e}_{j}^{\mathbf{T}} \\
\frac{\gamma_{0}-1}{2}\left(\pi+\tilde{b} \pi^{\nu}\right) \mathbf{e}_{j} & u_{j} I_{d}
\end{array}\right) .
$$

Consequently, $\partial_{t}(U-\tilde{U})+\sum_{j} a_{j}(U) \partial_{j}(U-\tilde{U})+\left(a_{j}(U)-a_{j}(\tilde{U})\right) \partial_{j} \tilde{U}=0$. Then, we make the scalar product with $(U-\tilde{U})$ and we integrate on $C_{T}$ for $T \in\left[0, T_{1}\right]$. We get

$$
\begin{aligned}
& \frac{1}{2} \int_{C_{T}}\left[\partial_{t}|U-\tilde{U}|^{2}+\sum_{j} \partial_{j}\left((U-\tilde{U}) \cdot a_{j}(U)(U-\tilde{U})\right)\right. \\
& \left.-\sum_{j}(U-\tilde{U}) \cdot \partial_{j}\left(a_{j}(U)\right)(U-\tilde{U})\right] \mathrm{d} x \mathrm{~d} t=-\int_{C_{T}} \sum_{j}(U-\tilde{U}) \cdot\left(a_{j}(U)-a_{j}(\tilde{U})\right) \partial_{j} \tilde{U} \mathrm{~d} x \mathrm{~d} t .
\end{aligned}
$$

Then using the Stokes formula and noting that $\partial C_{T}=\left(\{0\} \times B\left(x_{0}, R\right)\right) \cup\left(\{T\} \times B\left(x_{0}, R-M T\right)\right) \cup \mathcal{C}$, where $\mathcal{C}=\left\{(t, x) \in[0, T] \times B\left(x_{0}, R\right) \mid t=\frac{R-|x|}{M}\right\}$, we obtain

$$
\begin{aligned}
& \frac{1}{2} \int_{B\left(x_{0}, R-M T\right)}|U-\tilde{U}|^{2}(T, x) \mathrm{d} x-\frac{1}{2} \int_{B\left(x_{0}, \eta\right)}|U-\tilde{U}|^{2}(0, x) \mathrm{d} x \\
& +\frac{1}{2 \sqrt{1+1 / M^{2}}} \int_{\mathcal{C}}\left[|U-\tilde{U}|^{2}+\sum_{j}(U-\tilde{U}) \cdot a_{j}(U)(U-\tilde{U}) \frac{x_{j}}{M|x|}\right] \mathrm{d} \sigma \\
= & \int_{C_{T}}\left[\frac{1}{2} \sum_{j}(U-\tilde{U}) \cdot \partial_{j}\left(a_{j}(U)\right)(U-\tilde{U})-(U-\tilde{U}) \cdot\left(a_{j}(U)-a_{j}(\tilde{U})\right) \partial_{j} \tilde{U}\right] \mathrm{d} x \mathrm{~d} t .
\end{aligned}
$$

In addition

$$
\begin{gathered}
\left\|\partial_{j} a_{j}(U)\right\|_{\mathbf{L}^{\infty}} \leqslant C\|\mathrm{D} U\|_{\mathbf{L}^{\infty}\left(C_{T}\right)}\left(1+\|\pi\|_{\mathbf{L}^{\infty}\left(C_{T}\right)}^{\nu-1}\right), \\
\left|a_{j}(U)-a_{j}(\tilde{U})\right| \leqslant C|U-\tilde{U}|\left(1+\max \left(|\pi|^{\nu-1},|\tilde{\pi}|^{\nu-1}\right)\right) .
\end{gathered}
$$

Hence,

$$
\begin{aligned}
\int_{C_{T}} & \frac{1}{2}(U-\tilde{U}) \cdot \partial_{j}\left(a_{j}(U)\right)(U-\tilde{U})-(U-\tilde{U}) \cdot\left(a_{j}(U)-a_{j}(\tilde{U})\right) \partial_{j} \tilde{U} \mathrm{~d} x \mathrm{~d} t \\
\leqslant & C\left(\|\mathrm{D} U\|_{\mathbf{L}^{\infty}\left(C_{T}\right)}+\|\mathrm{D} \tilde{U}\|_{\mathbf{L}^{\infty}\left(C_{T}\right)}\right)\left(1+\max \left(\|\pi\|_{\mathbf{L}^{\infty}\left(C_{T}\right)}^{\nu-1},\|\tilde{\pi}\|_{\mathbf{L}^{\infty}\left(C_{T}\right)}^{\nu-1}\right)\right) \\
& \times \int_{0}^{T} \int_{B\left(x_{0}, R-M t\right)}|U-\tilde{U}|^{2} \mathrm{~d} x \mathrm{~d} t .
\end{aligned}
$$

Furthermore, the choice of $M$ implies

$$
\int_{\mathcal{C}}|U-\tilde{U}|^{2}+\sum_{j}(U-\tilde{U}) \cdot a_{j}(U)(U-\tilde{U}) \frac{x_{j}}{M|x|} \mathrm{d} \sigma \geqslant 0
$$

so finally we get the estimate

$$
\begin{aligned}
& \frac{1}{2} \int_{B\left(x_{0}, R-M T\right)}|U-\tilde{U}|^{2}(T, x) \mathrm{d} x-\frac{1}{2} \int_{B\left(x_{0}, \eta\right)}|U-\tilde{U}|^{2}(0, x) \mathrm{d} x \\
\leqslant & C\left(\|\mathrm{D} U\|_{\mathbf{L}^{\infty}\left(C_{T}\right)}+\|\left.\mathrm{D} \tilde{U}\right|_{\mathbf{L}^{\infty}\left(C_{T}\right)}\right)\left(1+\max \left(\|\pi\|_{\mathbf{L}^{\infty}\left(C_{T}\right)}^{\nu-1},\|\tilde{\pi}\|_{\mathbf{L}^{\infty}\left(C_{T}\right)}^{\nu-1}\right)\right) \\
& \int_{0}^{T} \int_{B\left(x_{0}, R-M t\right)}|U-\tilde{U}|^{2} \mathrm{~d} x \mathrm{~d} t .
\end{aligned}
$$


We conclude, thanks to the Gronwall lemma, that

$$
\frac{1}{2} \int_{B\left(x_{0}, R-M T\right)}|U-\tilde{U}|^{2}(T, x) \mathrm{d} x \leqslant \frac{1}{2} e^{C^{\prime} T} \int_{B\left(x_{0}, R\right)}\left|U_{0}-\tilde{U}_{0}\right|^{2}(x) \mathrm{d} x
$$

where $C^{\prime}=C\left(\|\mathrm{D} U\|_{\mathbf{L}^{\infty}\left(C_{T}\right)}+\|\mathrm{D} \tilde{U}\|_{\mathbf{L}^{\infty}\left(C_{T}\right)}\right)\left(1+\max \left(\|\pi\|_{\mathbf{L}^{\infty}\left(C_{T}\right)}^{\nu-1},\|\tilde{\pi}\|_{\mathbf{L}^{\infty}\left(C_{T}\right)}^{\nu-1}\right)\right)$, which is bounded under the hypotheses of the proposition.

\subsubsection{Local existence}

We construct a local solution of (3.1)-(3.2) such that the difference between this solution and $(0, \bar{u})$ is in $\mathscr{C}^{0}\left(\mathbb{R}^{+} ; \mathbf{H}^{m}\left(\mathbb{R}^{d} ; \mathbb{R}^{d+1}\right)\right) \cap \mathscr{C}^{1}\left(\mathbb{R}^{+} ; \mathbf{H}^{m-1}\left(\mathbb{R}^{d} ; \mathbb{R}^{d+1}\right)\right)$. The first step is the symmetrization of the system, given by Proposition 2.5. This result allows us to use a general theorem (see Theorem 2.6) giving the local existence of solution. Let us define, as above,

$$
\pi=2 \sqrt{\frac{\gamma_{0}}{\gamma_{0}-1}}\left(\frac{p}{\gamma_{0}-1}\right)^{\frac{\gamma_{0}-1}{2 \gamma_{0}}}=2 \sqrt{\frac{\gamma_{0}}{\gamma_{0}-1}}\left(\frac{\rho}{1-b \rho}\right)^{\frac{\gamma_{0}-1}{2}},
$$

where we assume that $0 \leqslant \rho<1 / b$. Then we use the same method as Grassin [7] to prove that the system (3.1) admits a local in time solution, with initial condition $u_{0}$ in the space $X$ and not in a Sobolev space (in particular, $u_{0}$ does not tend to 0 at infinity). Here we use the compactness of the support of $\rho_{0}$ (hypothesis (H4)) and the finite propagation speed of the solutions for a hyperbolic system. More precisely, we assume that $\operatorname{Supp}\left(\rho_{0}\right) \subset B(0, R)$ for $R>0$. Let $\eta>0$ and $\varphi \in \mathscr{C}_{c}^{\infty}\left(\mathbb{R}^{d} ; \mathbb{R}^{+}\right)$be such that $\varphi \equiv 1$ on $B(0, R+2 \eta)$. We obtain a local in time solution $\left(\pi^{\varphi}, u^{\varphi}\right)$ of problem (3.1) with initial conditions $\left(\pi_{0}, \varphi u_{0}\right) \in \mathbf{H}^{m}$ for $t \in[0, T[$. Propositions 2.7 and 2.8 ensure that the condition $0 \leqslant \rho<1 / b$ is satisfied.

Letting $\varepsilon \in] 0, T\left[\right.$, we introduce the maximal propagation speed $M=\sup \left\{\frac{\gamma_{0}-1}{2}\left(\left|\pi^{\varphi}\right|+\left|\pi^{\varphi}\right|^{\nu}\right)+\right.$ $\left.\left|u^{\varphi}\right| ; t \in[0, T-\varepsilon], x \in \mathbb{R}^{d}\right\}$. We also introduce $\left.\varepsilon^{\prime} \in\right] 0, \frac{\eta}{2 M}\left[\right.$ and $T_{1}=\min \left(T-\varepsilon, \frac{\eta}{2 M}-\varepsilon^{\prime}\right)$, the time for which this construction is available. We finally obtain a solution $(\pi, u)$ of (3.1) (3.2) by denoting

$$
(\pi, u)=\left\{\begin{array}{l}
\left(\pi^{\varphi}, u^{\varphi}\right) \text { in } \mathcal{K}, \\
(0, \bar{u}) \text { out of } \mathcal{K},
\end{array}\right.
$$

where $\mathcal{K}$ is the cone $\mathcal{K}=\left\{(t, x) ; 0 \leqslant t \leqslant T_{1}, x \in B(0, R+\eta+M t)\right\}$. Then it is sufficient to show that the solutions can be glued smoothly along $\partial \mathcal{K}$. Here we use the property of local uniqueness given by Proposition 3.2. Let indeed $x_{0} \in S(0, R+\eta)$ be in the sphere of radius $R+\eta$ of center 0 , and let $E_{x_{0}}=\left\{(t, x) ; t \in\left[0, T_{1}\right], x \in B\left(x_{0}, \eta-M t\right)\right\}$. The choice of $T_{1}$ implies in particular $\partial \mathcal{K} \subset \cup_{x_{0} \in S(0, R+\eta)} E_{x_{0}}$ (see Figure 1). The initial conditions of $\left(\pi^{\varphi}, u^{\varphi}\right)$ and $(0, \bar{u})$ coincide on $E_{x_{0}}$ since the support of $\pi_{0}$ is included in $B(0, R)$. Proposition 3.2 then allows us to claim that $\left(\pi^{\varphi}, u^{\varphi}\right)=(0, \bar{u})$ on $E_{x_{0}}$ and consequently on a neighborhood of $\partial \mathcal{K}$.

\subsubsection{Energy estimates}

When we compare it to the perfect polytropic gas case [7, we observe that the system (3.1) has an additional term, which will modify the estimates.

Let us denote $U=(\pi, u-\bar{u}), w=u-\bar{u}$, and $\bar{U}=(0, \bar{u})$. We also introduce

$$
A_{j}(U)=\left(\begin{array}{cc}
u_{j}-\bar{u}_{j} & \frac{\gamma_{0}-1}{2} \pi \mathbf{e}_{j}^{\mathbf{T}} \\
\frac{\gamma_{0}-1}{2} \pi \mathbf{e}_{j} & \left(u_{j}-\bar{u}_{j}\right) I_{d}
\end{array}\right), \quad B(\mathrm{D} \bar{U}, U)=\left(\begin{array}{c}
\frac{\gamma_{0}-1}{2} \pi \operatorname{div} \bar{u} \\
(w \cdot \nabla) \bar{u}
\end{array}\right),
$$

so that the system (3.1) can be written

$$
\partial_{t} U+\sum_{j=1}^{d} A_{j}(U) \partial_{j} U=-B(\mathrm{D} \bar{U}, U)-\sum_{j=1}^{d} \bar{u}_{j} \partial_{j} U-F(\mathrm{D} \bar{U}, \mathrm{D} U, U)
$$




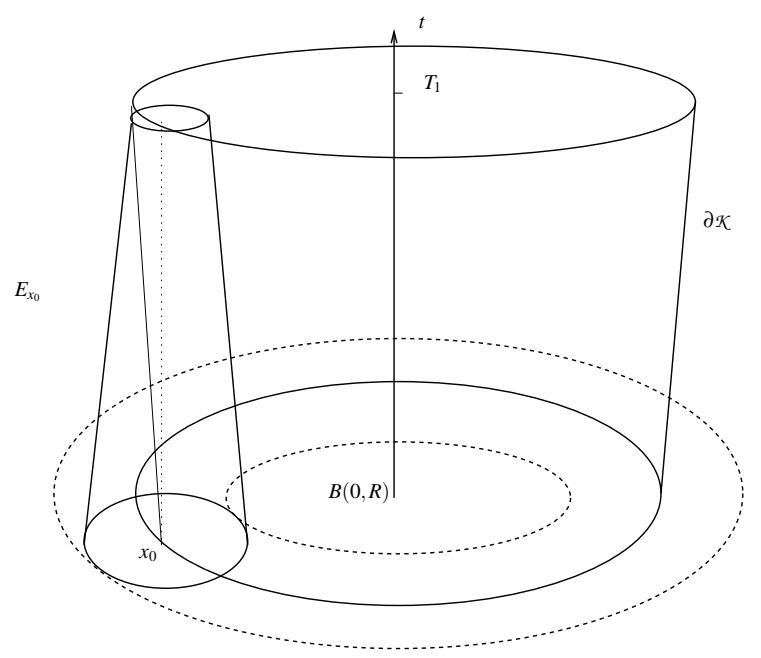

Figure 1: Gluing of the solutions along $\partial \mathcal{K}$.

where

$$
F(\mathrm{D} \bar{U}, \mathrm{D} U, U)=\frac{\gamma_{0}-1}{2} \tilde{b} \pi^{\nu}\left(\begin{array}{c}
\operatorname{div}(w+\bar{u}) \\
\nabla \pi
\end{array}\right), \quad \text { with } \quad \nu=\frac{\gamma_{0}+1}{\gamma_{0}-1} .
$$

Observing the properties of $\bar{u}$ described in Proposition 3.1, we expect the terms $\left\|\mathrm{D}^{k} U\right\|_{\mathbf{L}^{2}}$ for $k \in \llbracket 0, m \rrbracket$ to decrease with respect to time with a rate depending on $k$. Consequently, we introduce

$$
Y_{k}(t)=\left\|\mathrm{D}^{k} U(t, \cdot)\right\|_{\mathbf{L}^{2}}, \quad Z(t)=\sum_{k=0}^{m}(1+t)^{g_{k}} Y_{k}(t),
$$

with $g_{k}=k+c$, in which $c$ has to be chosen so that all the terms of $Z$ have the same decreasing in time. In order to estimate $Z$, we apply the operator $\mathrm{D}^{k}$ to (3.8), we make the scalar product with $\mathrm{D}^{k} U$, and we integrate on $\mathbb{R}^{d}$. The system (3.8) is different from the one considered by Grassin through the term $F(\mathrm{D} \bar{U}, \mathrm{D} U, U)$ defined in (3.9). We use now [7, Props. 3.2 and 3.3] to estimate the terms in common. We note these results here.

Proposition 3.3. Let $\alpha \in \mathbb{N}^{d}$ be a d-uplet of size $k \geqslant 0$ (that is, $|\alpha|_{1}=\alpha_{1}+\cdots+\alpha_{d}=k$ ). Let us denote, for $U=(\pi, u-\bar{u})$,

$$
\begin{aligned}
& R_{k}=-\int_{\mathbb{R}^{d}} \mathrm{D}^{k} U \cdot \mathrm{D}^{k}\left(\sum_{j=1}^{d} A_{j}(U) \partial_{j} U\right) \mathrm{d} x \\
& S_{k}=-\int_{\mathbb{R}^{d}} \mathrm{D}^{k} U \cdot \mathrm{D}^{k}\left(B(\mathrm{D} \bar{U}, U)+\sum_{j=1}^{d} \bar{u}_{j} \partial_{j} U\right) \mathrm{d} x ;
\end{aligned}
$$

then there exists a constant $C>0$ depending only on $m, d$ such that

$$
\left|R_{k}\right| \leqslant C(1+t)^{-g_{1}-d / 2} Y_{k}^{2} Z, \quad S_{k} \leqslant C(1+t)^{-g_{k}-2} Y_{k} Z-\frac{k+r}{1+t} Y_{k}^{2},
$$

where

$$
r=\min \left(1-\frac{d}{2},\left(\frac{\gamma_{0}}{2}-1\right) d\right)
$$


We have now to estimate $-\int_{\mathbb{R}^{d}} \mathrm{D}^{k} U \cdot \mathrm{D}^{k}(F(\mathrm{D} \bar{U}, \mathrm{D} U, U))$. Let us denote $\check{b}=\frac{\gamma_{0}-1}{2} \tilde{b}$ and

$$
I=-\check{b} \int_{\mathbb{R}^{d}} \mathrm{D}^{k} U \cdot \mathrm{D}^{k}\left(\pi^{\nu}\left(\begin{array}{c}
\operatorname{div} w \\
\nabla \pi
\end{array}\right)\right), \quad J=-\check{b} \int_{\mathbb{R}^{d}} \mathrm{D}^{k} U \cdot \mathrm{D}^{k}\left(\pi^{\nu}\left(\begin{array}{c}
\operatorname{div} \bar{u} \\
0
\end{array}\right)\right)
$$

so that $-\int_{\mathbb{R}^{d}} \mathrm{D}^{k} U \cdot \mathrm{D}^{k}(F(\mathrm{D} \bar{U}, \mathrm{D} U, U))=I+J$. A priori, $J$ is easier to estimate than $I$. However the estimate of $I$ is possible since the matrix $\pi^{\nu}\left(\begin{array}{cc}0 & \xi^{\mathbf{T}} \\ \xi & 0\end{array}\right)$ is symmetric.

a Estimate of $I$ We prove in section 4.1 the following lemma.

Lemma 3.4. With the notation introduced in section 3.1,

$$
|I| \leqslant C\|\pi\|_{\mathbf{L}^{\infty}}^{\nu-1}\|\mathrm{D} U\|_{\mathbf{L}^{\infty}}\left\|\mathrm{D}^{k} U\right\|_{\mathbf{L}^{2}}^{2} .
$$

b Estimate of $J$ Here, we divide $J$ into two parts: a first part $J_{1}$ which contains only first order derivatives of $\bar{u}$, and a second part $J_{2}$ in which all the derivatives of $\bar{u}$ are at least of order 2 . More precisely,

$$
\begin{aligned}
& J_{1}=-\check{b} \int_{\mathbb{R}^{d}} \sum_{\left.\alpha \in \mathbb{N}^{d}|| \alpha\right|_{1}=k}\left(\partial^{\alpha} \pi\right)\left(\partial^{\alpha} \pi^{\nu}\right) \operatorname{div} \bar{u}, \\
& J_{2}=J-J_{1} .
\end{aligned}
$$

For $J_{1}$, we use the first point of Proposition 3.1 giving the decreasing in time of $\mathrm{D} \bar{u}$, and Lemma 4.7 giving the estimate

$$
\left\|\partial^{\alpha}\left(\pi^{\nu}\right)\right\|_{\mathbf{L}^{2}} \leqslant C\|\pi\|_{\mathbf{L}^{\infty}}^{\nu-1}\left\|\mathrm{D}^{k} \pi\right\|_{\mathbf{L}^{2}},
$$

for any $d$-uplet $\alpha \in \mathbb{N}^{d}$ of size $k$, that is, satisfying $|\alpha|_{1}=\sum \alpha_{i}=k$.

We obtain, using the Cauchy-Schwarz inequality,

$$
\begin{aligned}
\left|J_{1}\right| & \leqslant \sum_{\alpha \in \mathbb{N}^{d} ;|\alpha|_{1}=k} \check{b}\left\|\partial^{\alpha} \pi\right\|_{\mathbf{L}^{2}}\left\|\partial^{\alpha}\left(\pi^{\nu}\right)\right\|_{\mathbf{L}^{2}}\|\operatorname{div} \bar{u}\|_{\mathbf{L}^{\infty}} \\
& \leqslant \frac{C}{1+t}\left\|\mathrm{D}^{k} U\right\|_{\mathbf{L}^{2}}^{2}\|\pi\|_{\mathbf{L}^{\infty}}^{\nu-1} .
\end{aligned}
$$

For $J_{2}$, we prove in section 4.1 the following lemma.

Lemma 3.5. With the notation introduced in section [3.1, there exists a constant $C>0$ such that

$$
\left|J_{2}\right| \leqslant C(1+t)^{d_{k}}\left\|\mathrm{D}^{k} U\right\|_{\mathbf{L}^{2}} Z^{\nu},
$$

where $d_{k}=\left(-g_{1}-\frac{d}{2}+1\right)(\nu-1)-g_{k}-2$.

c Reassembling of the estimates Assembling the results of Proposition 3.3 and the estimates (3.12) -(3.14) of $I$ and $J$, and finally using Lemma 4.5 we obtain

$$
\begin{aligned}
\frac{1}{2} \frac{\mathrm{d} Y_{k}^{2}}{\mathrm{~d} t}+\frac{k+r}{1+t} Y_{k}^{2} \leqslant & C(1+t)^{\beta} Y_{k}^{2} Z+C^{\prime}(1+t)^{-g_{k}-2} Y_{k} Z+C(1+t)^{\beta+(\beta+1)(\nu-1)} Y_{k}^{2} Z^{\nu} \\
& +C(1+t)^{(\beta+1)(\nu-1)-1} Y_{k}^{2} Z^{\nu-1}+C(1+t)^{(\beta+1)(\nu-1)-g_{k}-2} Y_{k} Z^{\nu},(3
\end{aligned}
$$

where we have denoted

$$
\beta=-g_{1}-\frac{d}{2}
$$


Here, we choose the constant $c$ introduced in $g_{k}=k+c$ in order to have $\beta=0$ and consequently a good decreasing in time. This means requiring that $g_{1}+d / 2=0$ and $c=-1-d / 2$. Consequently, we have

$$
g_{k}=k-\frac{d}{2}-1 .
$$

We now introduce

$$
a=1+d / 2+r>1
$$

so that $k+r=g_{k}+a$. We can now divide by $Y_{k}$ in (3.15), multiply by $(1+t)^{g_{k}}$, and sum on $k$ to obtain a differential inequality in $Z$ (defined in (3.10) $)$ :

$$
\frac{\mathrm{d} Z}{\mathrm{~d} t}+\frac{a}{1+t} Z \leqslant C\left(Z^{2}+\frac{Z}{(1+t)^{2}}+(1+t)^{\nu-1} Z^{\nu+1}\right) .
$$

Then, we introduce $\zeta(t)=(1+t)^{a} \exp \left(\frac{C}{1+t}\right) Z(t)$, and we deduce from the inequality just above that

$$
\frac{\mathrm{d} \zeta}{\mathrm{d} t} \leqslant \frac{C}{(1+t)^{a}}\left(\zeta^{2}+\zeta^{\nu+1}\right) .
$$

In addition, $\zeta^{2}+\zeta^{\nu+1} \leqslant 2 \zeta\left(1+\zeta^{\nu}\right)$ for $\nu \geqslant 2$. Therefore

$$
\frac{1}{\zeta\left(1+\zeta^{\nu}\right)} \frac{\mathrm{d} \zeta}{\mathrm{d} t} \leqslant \frac{C}{(1+t)^{a}}
$$

that is,

$$
\frac{\mathrm{d}}{\mathrm{d} t}(f(\zeta(t))) \leqslant \frac{C}{(1+t)^{a}},
$$

with $f(x)=\frac{1}{\nu} \ln \left(\frac{x^{\nu}}{1+x^{\nu}}\right)$. By integration, we obtain $f(\zeta(t))+\frac{C}{a-1}(1+t)^{-(a-1)} \leqslant f(\zeta(0))+\frac{C}{a-1}$. As $f$ is strictly increasing and one-by-one from $\mathbb{R}_{+}^{*}$ to $\mathbb{R}_{-}^{*}$, if $f(\zeta(0))+C /(a-1)$ belongs to the set on which $f^{-1}$ is well defined, we obtain

$$
\zeta(t) \leqslant f^{-1}(f(\zeta(0))+C /(a-1)) .
$$

But $f(\zeta(0))+C /(a-1) \leqslant 0$ is possible only if $\zeta(0)$ is small enough, since $f(x)$ tends to $-\infty$ when $x$ tends to 0 , and $\zeta(0)=\exp (C) Z(0)=\exp (C)\left\|\pi_{0}\right\|_{\mathbf{H}^{m}}$. The smallness condition is satisfied thanks to hypothesis $(\mathbf{H 1})$ with $0<\varepsilon_{0}<f^{-1}\left(\frac{-C}{a-1}\right)$.

d Conclusion We have obtained the following inequalities:

$$
\begin{aligned}
Z(t) & \leqslant \frac{1}{(1+t)^{a}} \exp \left(\frac{-C}{1+t}\right) f^{-1}\left(f\left(e^{C} Z(0)\right)+\frac{C}{a-1}\right), \\
Y_{k}(t) & \leqslant(1+t)^{-g_{k}} Z(t) \\
& \leqslant \frac{1}{(1+t)^{k+r}} \exp \left(\frac{-C}{1+t}\right) f^{-1}\left(f\left(e^{C} Z(0)\right)+\frac{C}{a-1}\right) .
\end{aligned}
$$

The $\mathbf{L}^{2}$ norms of the derivatives of the local solution $U$ consequently do not explode in finite time since $t \mapsto \frac{1}{(1+t)^{a}} \exp \left(\frac{-C}{1+t}\right)$ does not explode in finite time. Let us assume that the regular solution exists up to time $T$. Our estimates give us, for all $t \in\left[0, T\left[\right.\right.$, for $C_{T}$ not depending on $T$,

$$
\|(\pi, u-\bar{u})(t)\|_{\mathbf{H}^{m}} \leqslant C_{T} .
$$

Since Supp $\pi \subset B(0, R)$, our construction is possible when the norm of $(\pi, u)$ is bounded in $\mathbf{H}^{m}\left(\mathbb{R}^{d}\right)$ and

$$
\|(\pi, u)(t)\|_{\mathbf{H}^{m}(B(0, R))} \leqslant C_{T}+\|\bar{u}(t)\|_{\mathbf{H}^{m}(B(0, R))} \leqslant K_{T} .
$$

We can associate with the constant $K_{T}$ a time of existence $T_{*}\left(K_{T}\right)$ for the local in time solution. Let $\left.t_{1} \in\right] 0, T$ [ be such that $t \geqslant T-T_{*}\left(K_{T}\right)$. Introducing the solution with initial condition $\left(\pi\left(t_{1}\right), u\left(t_{1}\right)\right)$, we succeed in prolongating the solution up to time $T$, which finishes the proof. 


\subsection{General case}

\subsubsection{Local in time existence}

As in the isentropic case, we first seek to symmetrize the system. Let us denote

$$
\pi=\sqrt{\frac{\gamma_{0}-1}{\gamma_{0}}}\left(\frac{p}{\gamma_{0}-1}\right)^{\frac{\gamma_{0}-1}{2 \gamma_{0}}}=\sqrt{\frac{\gamma_{0}-1}{\gamma_{0}}}\left(\frac{\rho}{1-b \rho}\right)^{\frac{\gamma_{0}-1}{2}} \exp \left(\frac{\gamma_{0}-1}{2 \gamma_{0}} \frac{s}{c_{v}}\right) .
$$

The system (2.1) can be written in variables $(\pi, u, s)$ :

$$
\left\{\begin{aligned}
e^{s /\left(\gamma_{0} c_{v}\right)} \partial_{t} \pi+e^{s /\left(\gamma_{0} c_{v}\right)} u \cdot \nabla \pi+\frac{\gamma_{0}-1}{2} e^{s /\left(\gamma_{0} c_{v}\right)} \pi \operatorname{div} u & =-\frac{\gamma_{0}-1}{2} \tilde{b} \pi^{\frac{\gamma_{0}+1}{\gamma-1}} \operatorname{div} u, \\
\partial_{t} u+(u \cdot \nabla) u+\frac{\gamma_{0}-1}{2} e^{s /\left(\gamma_{0} c_{v}\right)} \pi \nabla \pi & =-\frac{\gamma_{0}-1}{2} \tilde{b} \pi^{\frac{\gamma_{0}+1}{\gamma_{0}-1}} \nabla \pi, \\
(1+t)^{-\theta}\left(\partial_{t} s+u \cdot \nabla s\right) & =0
\end{aligned}\right.
$$

where $\tilde{b}=b\left(\frac{\gamma_{0}-1}{4 \gamma_{0}}\right)^{\frac{1}{\gamma_{0}-1}}$. We introduce furthermore a parameter $\theta$ to be determined so that $(1+t)^{-\theta} s$ has a decreasing in time similar to those of the estimates obtained in the isentropic case.

In order to obtain local existence of a solution, we construct a solution by following the same strategy as in the isentropic case and using once again a property of local uniqueness, given by Proposition 3.6

\subsubsection{Local uniqueness}

Here we show a result similar to the one obtained in section 3.1 .1 in the isentropic case.

Proposition 3.6. Let $U_{0}=\left(\pi_{0}, u_{0}, s_{0}\right)^{\mathbf{T}}$ and $\tilde{U}_{0}$ be two initial data for (3.1). Let $U=(\pi, u, s)^{\mathbf{T}}$, $\tilde{U}$ be the two corresponding solutions defined for $0 \leqslant t \leqslant T_{0}$. Let $x_{0} \in \mathbb{R}^{d}$ and $R \geqslant 0$. We denote

$$
\begin{aligned}
M & =\sup \left\{e^{\frac{s}{2 \gamma_{0} c_{v}}}\left(\frac{\gamma_{0}-1}{2}|\pi|\left(1+\tilde{b}|\pi|^{\nu-1}\right)+|u|\right)(t, x),(t, x) \in\left[0, T_{0}\right] \times B\left(x_{0}, R\right)\right\}, \\
C_{T} & =\left\{(t, x) \in[0, T] \times B\left(x_{0}, R-M t\right)\right\} \quad \text { for } T \in\left[0, T_{1}\right],
\end{aligned}
$$

where $T_{1}=\min \left(T_{0}, R / M\right)$.

We assume that $U_{0} \in \mathbf{H}^{m}\left(\mathbb{R}^{d}\right), s_{0} \geqslant 0,\|\mathrm{D} \tilde{U}\|_{\mathbf{L}^{\infty}\left(\left[0, T_{0}\right] \times \mathbb{R}^{d}\right)}<\infty$, and that $\tilde{\pi}^{\nu-1} \in \mathbf{L}^{\infty}\left(C_{T}\right)$. Under these conditions, if $U_{0}=\tilde{U}_{0}$ on $B\left(x_{0}, R\right)$, then $U=\tilde{U}$ on $C_{T_{1}}$.

Proof. Let $U_{0}=\left(\pi_{0}, u_{0}, s_{0}\right), \tilde{U}_{0}=\left(\tilde{\pi}_{0}, \tilde{u}_{0}, \tilde{s}_{0}\right)$ be two initial data for (3.16) such that $U_{0} \in \mathbf{H}^{m}\left(\mathbb{R}^{d}\right)$. Let $U, \tilde{U}$ be the two solutions of the associated Cauchy problem. We assume that these solutions are defined on $\left[0, T_{0}\right]$ with $T_{0}>0$. Let furthermore $x_{0} \in \mathbb{R}^{d}, R \in \mathbb{R}_{+}^{*}$, and $M, C_{T}$ be as in (3.17) and (3.18). Then we have

$$
\alpha_{0}(U) \partial_{t} U+\sum_{j} \alpha_{j}(U) \partial_{j} U=0
$$

where $\alpha_{0}(U)=\operatorname{diag}\left(e^{\frac{s}{\gamma_{0} c v}}, 1, \ldots, 1,1\right) \in \mathcal{M}_{d+2}(\mathbb{R})$ and, for all $j \in\{1, \ldots, d\}$,

$$
\alpha_{j}(U)=\left(\begin{array}{ccc}
e^{\frac{s}{\gamma_{0} c_{v}}} u_{j} & e^{\frac{s}{\gamma_{0} c_{v}}} \frac{\gamma_{0}-1}{2}\left(\pi+\tilde{b} e^{\frac{-s}{\gamma_{0} c_{v}}} \pi^{\nu}\right) \mathbf{e}_{j}^{\mathbf{T}} & 0 \\
e^{\frac{s}{\gamma_{0} c_{v}}} \frac{\gamma_{0}-1}{2}\left(\pi+\tilde{b} e^{\frac{-s}{\gamma_{0} c_{v}}} \pi^{\nu}\right) \mathbf{e}_{j} & u_{j} I_{d} & 0 \\
0 & 0 & u_{J}
\end{array}\right) .
$$

We introduce, for $T \in\left[0, T_{1}\right]$,

$$
I=\int_{C_{T}}\left[\partial_{t}\left[(U-\tilde{U}) \cdot \alpha_{0}(U)(U-\tilde{U})\right]+\sum_{j} \partial_{j}\left[(U-\tilde{U}) \cdot \alpha_{j}(U)(U-\tilde{U})\right]\right] \mathrm{d} x \mathrm{~d} t
$$


We denote $|U-\tilde{U}|_{0}^{2}=(U-\tilde{U}) \alpha_{0}(U)(U-\tilde{U}),[U-\tilde{U}]_{t}=\int_{B\left(x_{0}, R-M t\right)}|(U-\tilde{U})(t, x)|_{0}^{2} \mathrm{~d} x$. We obtain from the Stokes formula

$$
\begin{aligned}
I= & \int_{\partial C_{T}}\left((U-\tilde{U}) \cdot \alpha_{0}(U)(U-\tilde{U}) n_{t}+\sum_{j}(U-\tilde{U}) \cdot \alpha_{j}(U)(U-\tilde{U}) n_{j}\right) \mathrm{d} \sigma \\
= & {[U-\tilde{U}]_{T}-[U-\tilde{U}]_{0} } \\
& +\frac{1}{\sqrt{1+1 / M^{2}}} \int_{\mathcal{C}}\left(|U-\tilde{U}|_{0}+\sum_{j}(U-\tilde{U}) \cdot \alpha_{j}(U)(U-\tilde{U}) \frac{x_{j}}{M|x|}\right) \mathrm{d} \sigma .
\end{aligned}
$$

But we also have

$$
\begin{aligned}
& \sum_{j}(U-\tilde{U}) \alpha_{j}(U)(U-\tilde{U}) \frac{x_{j}}{M|x|} \\
= & |U-\tilde{U}|_{0}^{2} \frac{u \cdot x}{M|x|}+2 e^{\frac{s}{\gamma_{0} c_{v}}} \frac{\gamma_{0}-1}{2}\left(\pi+\tilde{b} e^{\frac{-s}{\gamma_{0} c_{v}}} \pi^{\nu}\right)(\pi-\tilde{\pi}) \frac{(u-\tilde{u}) \cdot x}{M|x|} \\
\leqslant & |U-\tilde{U}|_{0}^{2} \frac{|u|}{M}+e^{s /\left(2 \gamma_{0} c_{v}\right)} \frac{\gamma_{0}-1}{2}\left(|\pi|+\tilde{b}|\pi|^{\nu}\right)\left(e^{s /\left(\gamma_{0} c_{v}\right)}|\pi-\tilde{\pi}|^{2}+|u-\tilde{u}|^{2}\right) \frac{1}{M} \\
\leqslant & \frac{1}{M}|U-\tilde{U}|_{0}^{2}\left(|u|+e^{s /\left(2 \gamma_{0} c_{v}\right)} \frac{\gamma_{0}-1}{2}\left(|\pi|+\tilde{b}|\pi|^{\nu}\right)\right) \\
\leqslant & |U-\tilde{U}|_{0}^{2} ;
\end{aligned}
$$

therefore

$$
I \geqslant[U-\tilde{U}]_{T}-[U-\tilde{U}]_{0}
$$

Besides, we have

$$
\alpha_{0}(U) \partial_{t}(U-\tilde{U})+\sum_{j} \alpha_{j}(U)(U-\tilde{U})=\sum_{j} \alpha_{0}(U)\left(\alpha_{0}(\tilde{U})^{-1} \alpha_{j}(\tilde{U})-\alpha_{0}(U)^{-1} \alpha_{j}(U)\right) \partial_{j} \tilde{U}
$$

thus

$$
\begin{aligned}
I= & \int_{C_{T}}(U-\tilde{U}) \partial_{t} \alpha_{0}(U)(U-\tilde{U})+\sum_{j}(U-\tilde{U}) \partial_{j} \alpha_{j}(U)(U-\tilde{U}) \\
& +2 \int_{C_{T}} \sum_{j}(U-\tilde{U}) \alpha_{0}(U)\left(\alpha_{0}(\tilde{U})^{-1} \alpha_{j}(\tilde{U})-\alpha_{0}(U)^{-1} \alpha_{j}(U)\right) \partial_{j} \tilde{U}
\end{aligned}
$$

Let us denote

$$
\begin{aligned}
& I_{1}=\int_{C_{T}}(U-\tilde{U}) \partial_{t} \alpha_{0}(U)(U-\tilde{U}), \\
& I_{2}=\sum_{j}(U-\tilde{U}) \partial_{j} \alpha_{j}(U)(U-\tilde{U}), \\
& I_{3}=2 \int_{C_{T}} \sum_{j}(U-\tilde{U}) \alpha_{0}(U)\left(\alpha_{0}(\tilde{U})^{-1} \alpha_{j}(\tilde{U})-\alpha_{0}(U)^{-1} \alpha_{j}(U)\right) \partial_{j} \tilde{U} .
\end{aligned}
$$

We obtain by computing explicitly $\partial_{t} \alpha_{0}$ and $\partial_{j} \alpha_{j}$

$$
I_{1}=-\int_{C_{T}} \frac{1}{\gamma_{0} c_{v}} e^{s /\left(\gamma_{0} c_{v}\right)}(\pi-\tilde{\pi})^{2}(u \cdot \nabla s)
$$


and

$$
\begin{aligned}
I_{2}= & \int_{C_{T}} \frac{1}{\gamma_{0} c_{v}} e^{s /\left(\gamma_{0} c_{v}\right)}(\pi-\tilde{\pi})^{2}(u \cdot \nabla s)+\int_{C_{T}} \operatorname{div}(u)|U-\tilde{U}|_{0}^{2} \\
& \quad+\int_{C_{T}}\left(\gamma_{0}-1\right) e^{s /\left(\gamma_{0} c_{v}\right)}(\pi-\tilde{\pi})(u-\tilde{u}) \cdot\left[\frac{1}{\gamma_{0} c_{v}} \pi \nabla s+\left(1+\tilde{b} e^{\frac{-s}{\gamma_{0} c_{v}}} \nu \pi^{\nu-1}\right) \nabla \pi\right] \\
\leqslant & -I_{1}+\|D U\|_{\mathbf{L}^{\infty}}\left(1+C\left(1+\|\pi\|_{\mathbf{L}^{\infty}}+\tilde{b} \nu\left\|\pi^{\nu-1}\right\|_{\mathbf{L}^{\infty}}\right)\right) \int_{0}^{T}[U-\tilde{U}]_{t} \mathrm{~d} t .
\end{aligned}
$$

Finally, we bound $I_{3}$ by computing

$$
\alpha_{0}(\tilde{U})^{-1} \alpha_{j}(\tilde{U})-\alpha_{0}(U)^{-1} \alpha_{j}(U)=-\left(\begin{array}{ccc}
u_{j}-\tilde{u}_{j} & \Psi_{1}(U) \mathbf{e}_{\mathbf{j}}^{\mathbf{T}} & 0 \\
\Psi_{2}(U) \mathbf{e}_{\mathbf{j}} & \left(u_{j}-\tilde{u}_{j}\right) I_{d} & 0 \\
0 & 0 & u_{j}-\tilde{u}_{j}
\end{array}\right),
$$

where

$$
\begin{aligned}
& \Psi_{1}(U)=\frac{\gamma_{0}-1}{2}\left(\pi-\tilde{\pi}+\tilde{b}\left(e^{\frac{-s}{\gamma_{0} c_{v}}} \pi^{\nu}-e^{\frac{-\tilde{s}}{\gamma_{0} c_{v}}} \tilde{\pi}^{\nu}\right)\right), \\
& \Psi_{2}(U)=\frac{\gamma_{0}-1}{2}\left(e^{\frac{s}{\gamma_{0} c_{v}}} \pi-e^{\frac{\tilde{s}}{\gamma_{0} c_{v}}} \tilde{\pi}+\tilde{b}\left(\pi^{\nu}-\tilde{\pi}^{\nu}\right)\right) .
\end{aligned}
$$

We denote $R=\max \left(\|s\|_{\mathbf{L}^{\infty}},\|\tilde{s}\|_{\mathbf{L}^{\infty}}\right)$. The exponential function being convex, we have

$$
\left|e^{s /\left(\gamma_{0} c_{v}\right)}-e^{\tilde{s} /\left(\gamma_{0} c_{v}\right)}\right| \leqslant 1 /\left(\gamma_{0} c_{v}\right) e^{R /\left(\gamma_{0} c_{v}\right)}|s-\tilde{s}| .
$$

So

$$
\begin{aligned}
e^{s /\left(\gamma_{0} c_{v}\right)} \pi-e^{\tilde{s} /\left(\gamma_{0} c_{v}\right)} \tilde{\pi} & \leqslant C(|\pi||s-\tilde{s}|+|\pi-\tilde{\pi}|), \\
e^{s /\left(\gamma_{0} c_{v}\right)} \pi^{\nu}-e^{\tilde{s} /\left(\gamma_{0} c_{v}\right)} \tilde{\pi}^{e} & \leqslant C \max (|\pi|,|\tilde{\pi}|)^{\nu-1}(|\pi||s-\tilde{s}|+|\pi-\tilde{\pi}|),
\end{aligned}
$$

which gives us

$$
\begin{aligned}
I_{3} \leqslant & \left(\|D \tilde{U}\|_{\mathbf{L}^{\infty}}+\|D U\|_{\mathbf{L}^{\infty}}\right)\left(1+C\left(1+\|\pi\|_{\mathbf{L}^{\infty}}\right)\left(1+\tilde{b} \nu \max \left(\|\pi\|_{\mathbf{L}^{\infty}}^{\nu-1},\|\tilde{\pi}\|_{\mathbf{L}^{\infty}}^{\nu-1}\right)\right)\right) \\
& \times \int_{0}^{T}[U-\tilde{U}]_{t} \mathrm{~d} t .
\end{aligned}
$$

Finally, we obtain

$$
[U-\tilde{U}]_{T}-[U-\tilde{U}]_{0} \leqslant C^{\prime} \int_{0}^{T}[U-\tilde{U}]_{t} \mathrm{~d} t
$$

where $C^{\prime}=\left(\|D \tilde{U}\|_{\mathbf{L}^{\infty}}+\|D U\|_{\mathbf{L}^{\infty}}\right)\left(1+C\left(1+\|\pi\|_{\mathbf{L}^{\infty}}\right)\left(1+\tilde{b} \nu \max \left(\|\pi\|_{\mathbf{L}^{\infty}}^{\nu-1},\|\tilde{\pi}\|_{\mathbf{L}^{\infty}}^{\nu-1}\right)\right)\right)$. We conclude, thanks to the Gronwall lemma, that

$$
\frac{1}{2} \int_{B\left(x_{0}, R-M T\right)}|U-\tilde{U}|^{2}(T, x) \mathrm{d} x \leqslant \frac{1}{2} e^{C^{\prime} T} \int_{B\left(x_{0}, R\right)}\left|U_{0}-\tilde{U}_{0}\right|^{2}(x) \mathrm{d} x
$$

\subsubsection{Estimates}

The system (3.16) can be written as

$$
A_{0} \partial_{t} V+\sum_{j=1}^{d} A_{j} \partial_{j} V=-B(\mathrm{D} \bar{V}, V)-\sum C_{j}(\bar{V}) \partial_{j} V-\frac{\gamma_{0}-1}{2} \tilde{b} \pi^{\nu}\left(\begin{array}{c}
\operatorname{div}(w+\bar{u}) \\
\nabla \pi \\
0
\end{array}\right)
$$


where $V=(\pi, u-\bar{u}, s) \in \mathbb{R}^{d+2}, \bar{V}=(0, \bar{u}, 0)$, and

$$
\begin{aligned}
A_{0} & =\operatorname{diag}\left(e^{s /\left(\gamma_{0} c_{v}\right)}, 1, \ldots, 1,(1+t)^{-\theta}\right) \in \mathcal{M}_{d+2}(\mathbb{R}), \\
C_{j} & =\bar{u}_{j} A_{0}, \\
A_{j} & =\left(\begin{array}{ccc}
e^{s /\left(\gamma_{0} c_{v}\right)} w_{j} & \frac{\gamma_{0}-1}{2} e^{s /\left(\gamma_{0} c_{v}\right)} \pi \mathbf{e}_{\mathbf{j}} \mathbf{T} & 0 \\
\frac{\gamma_{0}-1}{2} e^{s /\left(\gamma_{0} c_{v}\right)} \pi \mathbf{e}_{\mathbf{j}} & w_{j} I_{d} & 0 \\
0 & 0 & (1+t)^{-\theta} w_{j}
\end{array}\right), \\
B & =\left(\begin{array}{c}
\frac{\gamma_{0}-1}{2} e^{s /\left(\gamma_{0} c_{v}\right)} \pi \operatorname{div} \bar{u} \\
(w \cdot \nabla) \bar{u} \\
0
\end{array}\right) .
\end{aligned}
$$

We also introduce $N_{k}(t)=\left(\int_{\mathbb{R}^{d}} \mathrm{D}^{k} V \cdot A_{0}(V) \mathrm{D}^{k} V \mathrm{~d} x\right)^{1 / 2}$ and $Z(t)=\sum_{k=0}^{m}(1+t)^{g_{k}} N_{k}(t)$ with $g_{k}=k+r-a$ and $\left.\left.r=\theta / 2-d / 2, \theta \in\right] 0, \min \left(1, \frac{\gamma_{0}-1}{2}\right)\right]$. In order to obtain energy estimates, we apply $\mathrm{D}^{k}$ to (3.20) and we multiply it by $\mathrm{D}^{k} V$. Then, we integrate on $\mathbb{R}^{d}$. The additional term with respect to the perfect polytropic gases considered by Grassin [7] is now

$$
F^{*}(\mathrm{D} \bar{V}, \mathrm{D} V, V)=\frac{\gamma_{0}-1}{2} \tilde{b} \pi^{\nu}(\operatorname{div}(w+\bar{u}), \nabla \pi, 0) \in \mathbb{R}^{d+2} .
$$

With the notation $U=(\pi, w) \in \mathbb{R}^{d+1}$ and $F$ as in (3.9), the last component of $F^{*}$ being 0 , we have

$$
\int_{\mathbb{R}^{d}} \partial^{k} V \partial^{k}\left(F^{*}(\mathrm{D} \bar{V}, \mathrm{D} V, V)\right) \mathrm{d} x=\int_{\mathbb{R}^{d}} \partial^{k} U \partial^{k}(F(\mathrm{D} \bar{U}, \mathrm{D} U, U)) \mathrm{d} x .
$$

Using the estimates (3.12)-(3.14) we finally get an estimate on $Y_{k}$. Note that the definition of the norm $N_{k}$ is slightly different from the norm $Y_{k}$ introduced in the isentropic case; but if we introduce $v=\left(v_{1}, \ldots, v_{d+1}\right) \in \mathbb{R}^{d+1}, z \in \mathbb{R}$ and denote $v^{*}=\left(v_{1}, \ldots, v_{d+1}, z\right) \in \mathbb{R}^{d+2}$, then we have $\|v\|_{2} \leqslant e^{\left\|s_{0}\right\|_{\mathbf{L} \infty} /\left(\gamma_{0} c_{v}\right) t} v^{*} A_{0} v^{*}$. Consequently $Y_{k} \leqslant e^{\left\|s_{0}\right\|_{\mathbf{L} \infty} /\left(\gamma_{0} c_{v}\right)} N_{k}$, and the estimates on $Y_{k}$ obtained in the isentropic case give an estimate on $N_{k}$ in the general case.

Finally, we obtain, adding the estimate on $F$ obtained in the isentropic case to the estimates from Grassin in the general case:

$$
\begin{aligned}
\frac{1}{2} \frac{\mathrm{d} N_{k}^{2}}{\mathrm{~d} t}+\frac{k+r}{1+t} N_{k}^{2} \leqslant & C(1+t)^{\beta} N_{k}^{2} Z+C^{\prime}(1+t)^{-g_{k}-2} N_{k} Z+C(1+t)^{\beta+(\beta+1)(\nu-1)} N_{k}^{2} Z^{\nu} \\
& +C(1+t)^{(\beta+1)(\nu-1)-1} N_{k}^{2} Z^{\nu-1}+C(1+t)^{(\beta+1)(\nu-1)-g_{k}-2} N_{k} Z^{\nu} \\
& +C \sum_{\xi \in E_{k}} N_{k} Z^{2+\xi}(1+t)^{-g_{k}+\beta+\xi(\beta+1+\theta / 2)}
\end{aligned}
$$

where $\beta=-g_{1}-\frac{d}{2}$ and

$$
E_{k}=\llbracket 0, k \rrbracket \cup\left\{\frac{l}{k-1} ; l \in \llbracket 1, k-1 \rrbracket\right\} .
$$

Then, we choose $a$ so that $\beta=0$, i.e.

$$
\left.a=1+\theta / 2>1 \text { with } \theta \in] 0, \min \left(1, \frac{\gamma_{0}-1}{2}\right)\right] .
$$

Next, we simplify by $N_{k}$, we multiply by $(1+t)^{g_{k}}$ and we summate on $k$ to obtain

$$
\frac{\mathrm{d} Z}{\mathrm{~d} t}+\frac{a}{1+t} Z \leqslant C\left(Z^{2}+\frac{Z}{(1+t)^{2}}+(1+t)^{\nu-1} Z^{\nu+1}+Z^{2+m}(1+t)^{a m}\right) .
$$

We denote now $\zeta(t)=(1+t)^{a} \exp \left(\frac{C}{1+t}\right) Z(t)$ and we deduce from the inequality just above

$$
\frac{\mathrm{d} \zeta}{\mathrm{d} t} \leqslant \frac{C}{(1+t)^{a}}\left(\zeta^{2}+\zeta^{\nu+1}+\zeta^{m+2}\right)
$$

We conclude in the same way we did in the isentropic case, replacing $\nu$ by $\nu^{*}=\max (\nu, m+1) \geqslant 2$, since $\zeta^{2}+\zeta^{\nu+1}+\zeta^{m+2} \leqslant 2\left(\zeta^{2}+\zeta^{\nu^{*}+1}\right)$. 


\section{Technical tools}

Hereafter, we make use of the following notations for shortly denoting derivatives: $\partial^{i}$ with $i \in \mathbb{N}$ is used to mean any derivative $\partial^{\alpha}$ with $\alpha \in \mathbb{N}^{d}$ and $|\alpha|_{1}=\alpha_{1}+\ldots+\alpha_{d}=i ; \mathrm{D}^{i} U$ is used to denote the vector $\left(\partial^{\alpha} U\right)_{\alpha \in \mathbb{N}^{d} \|\left.\alpha\right|_{1}=i}$ of all derivatives of the given order $i$.

\subsection{Lemmas 3.4 and 3.5}

We show here Lemma 3.4, which states, with the notations introduced in section 3.1

$$
|I| \leqslant C\|\pi\|_{\mathbf{L}^{\infty}}^{\nu-1}\|\mathrm{D} U\|_{\mathbf{L}^{\infty}}\left\|\mathrm{D}^{k} U\right\|_{\mathbf{L}^{2}}^{2} .
$$

Proof of Lemma 3.4.

If $k=0, I=-\breve{b} \int_{\mathbb{R}^{d}} \pi^{\nu}(\pi \operatorname{div} w+w \cdot \nabla \pi) \mathrm{d} x$. By integration by parts, we obtain

$$
I=-\check{b} \frac{\nu}{\nu+1} \int_{\mathbb{R}^{d}} \pi^{\nu+1} \operatorname{div} w \operatorname{d} x \leqslant C\left\|\pi^{\nu-1}\right\|_{\mathbf{L}^{\infty}}\|\pi\|_{\mathbf{L}^{2}}^{2}\|\operatorname{div} w\|_{\mathbf{L}^{\infty}}
$$

If $k \geqslant 1, I$ is such that

$$
I=-\check{b} \sum_{\left.\alpha \in \mathbb{N}^{d}|| \alpha\right|_{1}=k}\left[\int_{\mathbb{R}^{d}} \partial^{\alpha} \pi \partial^{\alpha}\left(\pi^{\nu} \operatorname{div} w\right)+\sum_{j} \partial^{\alpha} w_{j} \partial^{\alpha}\left(\pi^{\nu} \partial_{j} \pi\right)\right] \mathrm{d} x .
$$

Expanding, we find

$$
I=-\check{b} \sum_{\left.\alpha \in \mathbb{N}^{d}|| \alpha\right|_{1}=k} \int_{\mathbb{R}^{d}}\left[\partial^{\alpha}(\pi) \pi^{\nu} \partial^{\alpha}(\operatorname{div} w)+\sum_{j} \partial^{\alpha}\left(w_{j}\right) \pi^{\nu} \partial^{\alpha}\left(\partial_{j} \pi\right)+\Sigma\right] \mathrm{d} x,
$$

where $\Sigma$ is a sum of terms as $\int_{\mathbb{R}^{d}} \partial^{\alpha} U \partial^{\alpha_{1}}\left(\pi^{\nu}\right) \partial^{\alpha_{2}} U$, where $U$ is any of its components and $\left|\alpha_{1}\right|_{1}=l,\left|\alpha_{2}\right|_{1}=k+1-l$, with $l \in \llbracket 1, k \rrbracket$, so that the derivatives are of order less than or equal to $k$. We first treat the first two terms of the sum (4.1) for a $d$-uplet $\alpha \in \mathbb{N}^{d}$, of size $k$, that is, $|\alpha|_{1}=\alpha_{1}+\cdots+\alpha_{d}=k$. By integration by parts, we find

$$
\begin{aligned}
& \int_{\mathbb{R}^{d}} \partial^{\alpha}(\pi) \pi^{\nu} \partial^{\alpha}(\operatorname{div} w)+\sum_{j} \partial^{\alpha}\left(w_{j}\right) \pi^{\nu} \partial^{\alpha}\left(\partial_{j} \pi\right) \\
= & \int_{\mathbb{R}^{d}} \pi^{\nu} \sum_{j} \partial_{j}\left(\partial^{\alpha}(\pi) \partial^{\alpha}\left(w_{j}\right)\right) \\
= & -\sum_{j} \int_{\mathbb{R}^{d}} \partial_{j}\left(\pi^{\nu}\right) \partial^{\alpha}(\pi) \partial^{\alpha}\left(w_{j}\right) .
\end{aligned}
$$

Hence, $I$ is a sum of terms as $\int \partial^{k} U \partial^{l}\left(\pi^{\nu}\right) \partial^{k-l+1} U$; where $1 \leqslant l \leqslant k$.

Note that here we used the notation in which $\partial^{k} U$ means $\partial^{\alpha} U$ for a given $\alpha \in \mathbb{N}^{d}$ such that $|\alpha|_{1}=\sum \alpha_{j}=k$. Furthermore, we write $U$ for any of its components. Consequently, $\partial^{k} U$ can mean $\partial^{\alpha} w_{j}$. We will use this notation from now on.

If $k \geqslant 1$ and $l \neq 1, l \neq k$, using the Hölder inequality and the Gagliardo-Nirenberg lemma 
(see Lemma 4.1) we have

$$
\begin{gathered}
\int_{\mathbb{R}^{d}} \partial^{k} U \partial^{l}\left(\pi^{\nu}\right) \partial^{k-l+1} U \mathrm{~d} x \leqslant C\left\|\partial^{k} U\right\|_{\mathbf{L}^{2}}\left\|\partial^{l}\left(\pi^{\nu}\right) \partial^{k-l+1} U\right\|_{\mathbf{L}^{2}} \\
\leqslant C\left\|\partial^{k} U\right\|_{\mathbf{L}^{2}}\left\|\partial^{l}\left(\pi^{\nu}\right)\right\|_{\mathbf{L}^{2 \frac{k-1}{l-1}}}\left\|\partial^{k-l+1} U\right\|_{\mathbf{L}^{2 \frac{k-1}{k-l}}} \\
\leqslant C\left\|\partial^{k} U\right\|_{\mathbf{L}^{2}}\left\|\partial\left(\pi^{\nu}\right)\right\|_{\mathbf{L}^{\infty} \frac{-\frac{l-1}{k-1}}{k}\left\|\partial^{k}\left(\pi^{\nu}\right)\right\|_{\mathbf{L}^{2}}^{\frac{l-1}{k-1}}} \\
\times\|\partial U\|_{\mathbf{L}^{\infty}}^{1-\frac{k-l}{k-1}}\left\|\partial^{k} U\right\|_{\mathbf{L}^{2}}^{\frac{k-l}{k-1}}
\end{gathered}
$$

We use next Lemma 4.7 and the inequality

$$
\left\|\partial\left(\pi^{\nu}\right)\right\|_{\mathbf{L}^{\infty}}=\left\|\nu \pi^{\nu-1} \partial \pi\right\|_{\mathbf{L}^{\infty}} \leqslant C\|\pi\|_{\mathbf{L}^{\infty}}^{\nu-1}\|\partial \pi\|_{\mathbf{L}^{\infty}}
$$

to obtain

$$
\int_{\mathbb{R}^{d}} \partial^{k} U \partial^{l}\left(\pi^{\nu}\right) \partial^{k-l+1} U \mathrm{~d} x \leqslant C\left\|\partial^{k} U\right\|_{\mathbf{L}^{2}}^{2}\|\partial U\|_{\mathbf{L}^{\infty}}\|\pi\|_{\mathbf{L}^{\infty}}^{\nu-1} .
$$

If $k \geqslant 1$ and $l=k$, we have to estimate

$$
\begin{aligned}
\int_{\mathbb{R}^{d}} \partial^{k} U \partial^{k}\left(\pi^{\nu}\right) \partial U & \leqslant\left\|\partial^{k} U\right\|_{\mathbf{L}^{2}}\left\|\partial^{k}\left(\pi^{\nu}\right)\right\|_{\mathbf{L}^{2}}\|\partial U\|_{\mathbf{L}^{\infty}} \\
& \leqslant C\left\|\partial^{k} U\right\|_{\mathbf{L}^{2}}\|\pi\|_{\mathbf{L}^{\infty}}^{\nu-1}\left\|\partial^{k} \pi\right\|_{\mathbf{L}^{2}}\|\partial U\|_{\mathbf{L}^{\infty}} .
\end{aligned}
$$

If $k \geqslant 1$ and $l=1$, we have to estimate

$$
\int_{\mathbb{R}^{d}} \partial^{k} U \partial\left(\pi^{\nu}\right) \partial^{k} U \leqslant\left\|\partial^{k} U\right\|_{\mathbf{L}^{2}}^{2}\left\|\partial^{1}\left(\pi^{\nu}\right)\right\|_{\mathbf{L}^{\infty}} .
$$

The inequality $\left\|\partial^{1}\left(\pi^{\nu}\right)\right\|_{\mathbf{L}^{\infty}} \leqslant C\|\pi\|_{\mathbf{L}^{\infty}}^{\nu-1}\left\|\partial^{1} \pi\right\|_{\mathbf{L}^{\infty}}$ allows us to conclude the proof.

We prove now Lemma 3.5, which states, with the notation introduced in section 3.1

$$
J_{2} \leqslant C(1+t)^{d_{k}}\left\|\mathrm{D}^{k} U\right\|_{\mathbf{L}^{2}} Z^{\nu},
$$

where $d_{k}=(\beta+1)(\nu-1)-g_{k}-2$ and $\beta=-g_{1}-\frac{d}{2}$.

Proof of Lemma 3.5. For $k=0, J_{2}=0$. We consider here $k \geqslant 1 ; J_{2}$ is then a sum of terms $\int \partial^{k} U \partial^{l}\left(\pi^{\nu}\right) \partial^{k-l+1} \bar{u} \mathrm{~d} x$ for $0 \leqslant l \leqslant k-1$. The choice of initial conditions gives us $U \in \mathbf{H}^{m}$, but we do not know if $\mathrm{D}^{2} U \in \mathbf{L}^{\infty}$. So we can distinguish two cases: $m>2+d / 2$ and $\mathrm{D}^{2} U \in \mathbf{L}^{\infty}$, or $m \leqslant 2+d / 2$.

Case $m>2+d / 2$. We now study different cases, according to the values of $k$ and $l$.

1. If $k \geqslant 1$ and $l=0$, we use Proposition 3.1 and Lemma 4.5 to obtain the estimate

$$
\begin{aligned}
\int_{\mathbb{R}^{d}} \pi^{\nu} \partial^{k} \pi \partial^{k+1} \bar{u} \mathrm{~d} x & \leqslant\left\|\pi^{\nu}\right\|_{\mathbf{L}^{\infty}}\left\|\partial^{k} U\right\|_{\mathbf{L}^{2}}\left\|\partial^{k+1} \bar{u}\right\|_{\mathbf{L}^{2}} \\
& \leqslant C(1+t)^{(\beta+1) \nu} Z^{\nu}\left\|\mathrm{D}^{k} U\right\|_{\mathbf{L}^{2}}(1+t)^{d / 2-k-2} \\
& \leqslant C(1+t)^{d_{k}}\left\|\mathrm{D}^{k} U\right\|_{\mathbf{L}^{2}} Z^{\nu}
\end{aligned}
$$

since $k-d / 2+2=\beta+1+g_{k}+2$, we are done. 
2. If $k \geqslant 2$ and $l=1$, we have to estimate

$$
\begin{aligned}
\int_{\mathbb{R}^{d}} \pi^{\nu-1} \partial \pi \partial^{k} \pi \partial^{k} \bar{u} \mathrm{~d} x & \leqslant\left\|\partial^{k} \pi\right\|_{\mathbf{L}^{2}}\left\|\partial^{k} \bar{u}\right\|_{\mathbf{L}^{2}}\|\pi\|_{\mathbf{L}^{\infty}}^{\nu-1}\|\partial \pi\|_{\mathbf{L}^{\infty}} \\
& \leqslant C(1+t)^{d / 2-k-1}(1+t)^{(\beta+1)(\nu-1)+\beta} Z^{\nu}\left\|\partial^{k} U\right\|_{\mathbf{L}^{2}},
\end{aligned}
$$

which concludes that case, since $d / 2-k-1+\beta+(\beta+1)(\nu-1)=(\beta+1)(\nu-1)-g_{k}-2$.

3. If $k=3$ and $l=2$, we use Proposition 3.1 and Lemmas 4.5 and 4.7 to obtain the estimate

$$
\begin{aligned}
\int_{\mathbb{R}^{d}} \partial^{3} \pi \partial^{2}\left(\pi^{\nu}\right) \partial^{2} \bar{u} \mathrm{~d} x & \leqslant\left\|\partial^{k} \pi\right\|_{\mathbf{L}^{2}}\left\|\partial^{2} \bar{u}\right\|_{\mathbf{L}^{\infty}}\left\|\partial^{2}\left(\pi^{\nu}\right)\right\|_{\mathbf{L}^{2}} \\
& \leqslant(1+t)^{-3+(\beta+1)(\nu-1)-g_{2}}\left\|\partial^{k} U\right\|_{\mathbf{L}^{2}} Z^{\nu},
\end{aligned}
$$

and we are done as $g_{2}+3=g_{3}+2$.

4. If $k>3$ and $l \in \llbracket 2, k-1 \rrbracket$, we use Lemma 4.2. Denoting $q=2 \frac{k-3}{l-2}$ and $q^{\prime}=2 \frac{k-3}{k-l-1}$ so that $1 / q+1 / q^{\prime}=1 / 2$, we obtain

$$
\begin{aligned}
& \int_{\mathbb{R}^{d}} \partial^{k} \pi \partial^{l}\left(\pi^{\nu}\right) \partial^{k-l+1} \bar{u} \mathrm{~d} x \\
\leqslant & \left\|\partial^{k} \pi\right\|_{\mathbf{L}^{2}}\left\|\partial^{l}\left(\pi^{\nu}\right)\right\|_{\mathbf{L}^{q}}\left\|\partial^{k-l+1} \bar{u}\right\|_{\mathbf{L}^{q^{\prime}}} \\
\leqslant & \left\|\partial^{k} \pi\right\|_{\mathbf{L}^{2}}\left\|\mathrm{D}^{2}\left(\pi^{\nu}\right)\right\|_{\mathbf{L}^{\infty}}^{1-2 / q}\left\|\mathrm{D}^{k-1}\left(\pi^{\nu}\right)\right\|_{\mathbf{L}^{2}}^{2 / q}\left\|\mathrm{D}^{2} \bar{u}\right\|_{\mathbf{L}^{\infty}}^{1-2 / q^{\prime}}\left\|\mathrm{D}^{k-1} \bar{u}\right\|_{\mathbf{L}^{2}}^{2 / q^{\prime}} .
\end{aligned}
$$

Since

$$
\left\|\mathrm{D}^{2} \bar{u}\right\|_{\mathbf{L}^{\infty}} \leqslant C(1+t)^{-3}, \quad \quad\left\|\mathrm{D}^{k-1} \bar{u}\right\|_{\mathbf{L}^{2}} \leqslant C(1+t)^{d / 2-k},
$$

and

$$
\begin{aligned}
\left\|\mathrm{D}^{2}\left(\pi^{\nu}\right)\right\|_{\mathbf{L}^{\infty}} & \leqslant C\left(\|\pi\|_{\mathbf{L}^{\infty}}^{\nu-2}\|\mathrm{D} \pi\|_{\mathbf{L}^{\infty}}^{2}+\|\pi\|_{\mathbf{L}^{\infty}}^{\nu-1}\left\|\mathrm{D}^{2} \pi\right\|_{\mathbf{L}^{\infty}}\right) \\
& \leqslant C(1+t)^{(\beta+1) \nu-2} Z^{\nu}, \\
\left\|\mathrm{D}^{k-1}\left(\pi^{\nu}\right)\right\|_{\mathbf{L}^{2}} & \leqslant C\|\pi\|_{\mathbf{L}^{\infty}}^{\nu-1}\left\|\mathrm{D}^{k-1} \pi\right\|_{\mathbf{L}^{2}} \\
& \leqslant C(1+t)^{(\beta+1)(\nu-1)-g_{k}+1} Z^{\nu},
\end{aligned}
$$

we obtain

$$
J_{2} \leqslant C(1+t)^{m_{k}}\left\|\mathrm{D}^{k} \pi\right\|_{\mathbf{L}^{2}} Z^{\nu}
$$

where

$$
\begin{aligned}
m_{k}= & \left(1-\frac{2}{q}\right)((\beta+1) \nu-2)+\frac{2}{q}\left((\beta+1)(\nu-1)-g_{k}+1\right)-3\left(1-\frac{2}{q^{\prime}}\right) \\
& +\frac{2}{q^{\prime}}\left(\frac{d}{2}-k\right) \\
= & (\beta+1)(\nu-1)-g_{k}-2 \\
= & d_{k} .
\end{aligned}
$$


Case $m \leqslant 2+d / 2$.

First, we note that the computations of the cases $k \geqslant 1$ and $l=0, k \geqslant 2$ and $l=1$, and $k=3$ and $l=2$ are similar. There remains to treat the case $k>3$ and $2 \leqslant l \leqslant k-1$. Since $k \leqslant m \leqslant 2+d / 2$, we have necessarily $k \leqslant 3$ if $d=1,2$, or 3 , and we are done.

We assume now $d \geqslant 4$. Let us denote $h=\frac{1}{2}(k+1+d / 2)>2$. Then we have $h \leqslant m$ and

$$
0<h-l \leqslant \frac{d-1}{2}, \quad \frac{1}{2} \leqslant h-(k+1-l) \leqslant \frac{d-1}{2} .
$$

We introduce $h_{1}=h-l, h_{2}=h-(k-l+1)$ and $1 / q_{1}=1 / 2-h_{1} / d, 1 / q_{2}=1 / 2-h_{2} / d$. Therefore $1 / q_{1}+1 / q_{2}=1 / 2$, which allows us to use the Hölder inequality

$$
J_{2}(k, l)=\int_{\mathbb{R}^{d}} \partial^{k} \pi \partial^{l}\left(\pi^{\nu}\right) \partial^{k-l+1} \bar{u} \mathrm{~d} x \leqslant\left\|\partial^{k} \pi\right\|_{\mathbf{L}^{2}}\left\|\partial^{l}\left(\pi^{\nu}\right)\right\|_{\mathbf{L}^{q_{1}}}\left\|\partial^{k-l+1} \bar{u}\right\|_{\mathbf{L}^{q_{2}}} .
$$

Next, we apply Lemma 4.3 to find

$$
J_{2}(k, l) \leqslant C\left\|\partial^{k} \pi\right\|_{\mathbf{L}^{2}}\left\|\mathrm{D}^{h}\left(\pi^{\nu}\right)\right\|_{\mathbf{L}^{2}}\left\|\mathrm{D}^{h} \bar{u}\right\|_{\mathbf{L}^{2}} .
$$

Finally, we use Lemma 4.7 and Proposition 3.1 to obtain

$$
J_{2}(k, l) \leqslant C\left\|\partial^{k} U\right\|_{\mathbf{L}^{2}}(1+t)^{d / 2-h-1-g_{h}+(\beta+1)(\nu-1)} Z^{\nu} .
$$

As $d / 2-h-1-g_{h}+(\beta+1)(\nu-1)=(\beta+1)(\nu-1)-g_{k}-2$, we are done.

\subsection{The Gagliardo-Nirenberg inequality and its consequences}

\subsubsection{The Gagliardo-Nirenberg inequality}

Lemma 4.1 (Gagliardo-Nirenberg). (See [23, Prop. 3.5, p. 4]). Let $r>0, i \in[0, r]$, and $z \in\left(\mathbf{L}^{\infty} \cap \mathbf{H}^{r}\right)\left(\mathbb{R}^{d}\right)$. Then $\partial^{i} z \in \mathbf{L}^{2 r / i}\left(\mathbb{R}^{d}\right)$ and

$$
\left\|\partial^{i} z\right\|_{\mathbf{L}^{2 r / i}} \leqslant C_{i, r}\|z\|_{\mathbf{L}^{\infty}}^{1-i / r}\left\|\mathrm{D}^{r} z\right\|_{\mathbf{L}^{2}}^{i / r} .
$$

We deduce easily from Lemma 4.1 the following result.

Lemma 4.2. Let $z \in \mathbf{H}^{m}$ be such that $\mathrm{D}^{2} z \in \mathbf{L}^{\infty}$; then for all $k \in[4, m]$, for all $i \in[2, k]$, we have $\mathrm{D}^{i} z \in \mathbf{L}^{q}$ for $q=2 \frac{k-3}{i-2}$ and

$$
\left\|\partial^{i} z\right\|_{\mathbf{L}^{q}} \leqslant C\left\|\mathrm{D}^{2} z\right\|_{\mathbf{L}^{\infty}}^{1-2 / q}\left\|\mathrm{D}^{k-1} z\right\|_{\mathbf{L}^{2}}^{2 / q} .
$$

Thanks to the Sobolev imbedding (see [23, p. 4]) and Lemma 4.1 we also prove the following lemma.

Lemma 4.3. Let $\ell \in] 0, d / 2[$ and $1 / q=1 / 2-\ell / d$. There exists $C>0$ depending on $\ell, q, d$ such that for all $z \in \mathbf{H}^{\ell}\left(\mathbb{R}^{d}\right)$ we have

$$
\|z\|_{\mathbf{L}^{q}} \leqslant C\left\|\mathrm{D}^{\ell} z\right\|_{\mathbf{L}^{2}}
$$

Proof. The space $\mathbf{H}^{\ell}\left(\mathbb{R}^{d}\right)$ is endowed with the norm $\|\cdot\|_{\mathbf{L}^{2}}+\left\|\mathrm{D}^{\ell} \cdot\right\|_{\mathbf{L}^{2}}$. The Sobolev imbedding between $\mathbf{H}^{\ell}$ and $\mathbf{L}^{q}$ can then be written, for a given $C>0$, as

$$
\|z\|_{\mathbf{L}^{q}} \leqslant C\left(\|z\|_{\mathbf{L}^{2}}+\left\|\mathrm{D}^{\ell} z\right\|_{\mathbf{L}^{2}}\right), \quad \text { for all } z \in \mathbf{H}^{\ell}
$$


Let us define now, for $z \in \mathbf{H}^{\ell}, \lambda \in \mathbb{R}^{+}$, the function $z_{\lambda} \in \mathbf{H}^{\ell}$ such that $z_{\lambda}(x)=z(\lambda x)$. Applying (4.2) to $z_{\lambda}$ and noting that

$$
\left\|z_{\lambda}\right\|_{\mathbf{L}^{q}}=\lambda^{\frac{-d}{q}}\|z\|_{\mathbf{L}^{q}}, \quad\left\|\mathrm{D}^{\ell} z_{\lambda}\right\|_{\mathbf{L}^{2}}=\lambda^{\ell-\frac{d}{2}}\left\|\mathrm{D}^{\ell} z\right\|_{\mathbf{L}^{2}},
$$

we obtain

$$
\|z\|_{\mathbf{L}^{q}} \leqslant C \lambda^{\frac{d}{q}-\frac{d}{2}}\left(\|z\|_{\mathbf{L}^{2}}+\lambda^{\ell}\left\|\mathrm{D}^{\ell} z\right\|_{\mathbf{L}^{2}}\right)
$$

where, by definition, $\frac{d}{q}-\frac{d}{2}=-\ell$. Consequently, introducing $\lambda=\left(\frac{\|z\|_{\mathrm{L}^{2}}}{\left\|\mathrm{D}^{\ell} z\right\|_{\mathrm{L}^{2}}}\right)^{1 / \ell}$, we have

$$
\|z\|_{\mathbf{L}^{q}} \leqslant 2 C\left\|\mathrm{D}^{\ell} z\right\|_{\mathbf{L}^{2}}
$$

Similarly, we prove the following lemma.

Lemma 4.4. Let $p>d / 2$. There exists $C>0$ such that for all $z \in \mathbf{H}^{p}\left(\mathbb{R}^{d}\right)$

$$
\|z\|_{\mathbf{L}^{\infty}} \leqslant C\|z\|_{\mathbf{L}^{2}}^{1-\frac{d}{2 p}}\left\|\mathrm{D}^{p} z\right\|_{\mathbf{L}^{2}}^{\frac{d}{2 p}}
$$

Proof. We now use the continuous imbedding $\mathbf{H}^{p}\left(\mathbb{R}^{d}\right) \subset \mathbf{L}^{\infty}\left(\mathbb{R}^{d}\right)$. Thus, there exists $C>0$ such that

$$
\|z\|_{\mathbf{L}^{\infty}} \leqslant C\left(\|z\|_{\mathbf{L}^{2}}+\left\|\mathrm{D}^{p} z\right\|_{\mathbf{L}^{2}}\right) \quad \text { for all } z \in \mathbf{H}^{p} .
$$

Applying the inequality to $z_{\lambda}: x \mapsto z(\lambda x)$, we obtain, since $z_{\lambda}$ satisfies $\left\|z_{\lambda}\right\|_{\mathbf{L}^{\infty}}=\|z\|_{\mathbf{L}^{\infty}}$ and (4.3),

$$
\|z\|_{\mathbf{L}^{\infty}} \leqslant C \lambda^{\frac{-d}{2}}\left(\|z\|_{\mathbf{L}^{2}}+\lambda^{p}\left\|\mathrm{D}^{p} z\right\|_{\mathbf{L}^{2}}\right) .
$$

Taking $\lambda=\left(\frac{\|z\|_{\mathbf{L}^{2}}}{\left\|\mathrm{D}^{p} z\right\|_{\mathbf{L}^{2}}}\right)^{1 / p}$, we have finished the proof.

\subsubsection{Estimates}

Lemma 4.5. Let $m>1+d / 2, U \in \mathbf{H}^{m}\left(\mathbb{R}^{d}\right), r, a \in \mathbb{R}$, and $Z$ be the norm defined by (3.10):

$$
Z(t)=\sum_{k=0}^{m}(1+t)^{g_{k}}\left\|\mathrm{D}^{k} U(t)\right\|_{\mathbf{L}^{2}},
$$

with $g_{k}=k+r-a$. Then we have the following:

1. $\|U(t)\|_{\mathbf{L}^{\infty}} \leqslant C(1+t)^{\beta+1} Z(t)$.

2. $\|\mathrm{D} U(t)\|_{\mathbf{L}^{\infty}} \leqslant C(1+t)^{\beta} Z(t)$.

3. If $m>2+d / 2$, then $\left\|\mathrm{D}^{2} U(t)\right\|_{\mathbf{L}^{\infty}} \leqslant C(1+t)^{\beta-1} Z(t)$.

With $\beta=-g_{1}-d / 2$.

Proof. 1. Applying Lemma 4.4 to $U$, we obtain

$$
\|U\|_{\mathbf{L}^{\infty}} \leqslant C\|U\|_{\mathbf{L}^{2}}^{1-\frac{d}{2 m}}\left\|\mathrm{D}^{m} U\right\|_{\mathbf{L}^{2}}^{\frac{d}{2 m}} .
$$


But we also have

$$
\|U\|_{\mathbf{L}^{2}} \leqslant(1+t)^{-g_{0}} Z, \quad\left\|\mathrm{D}^{m} U\right\|_{\mathbf{L}^{2}} \leqslant(1+t)^{-g_{m}} Z,
$$

so

$$
\|U\|_{\mathbf{L}^{\infty}} \leqslant C(1+t)^{s} Z,
$$

where $s=-g_{0}\left(1-\frac{d}{2 m}\right)-\left(g_{0}+m\right) \frac{d}{2 m}=-g_{0}-\frac{d}{2}=\beta+1$.

2. Applying Lemma 4.4 to $\mathrm{D} U$, with $p=m-1$, we have

$$
\|\mathrm{D} U\|_{\mathbf{L}^{\infty}} \leqslant C\|\mathrm{D} U\|_{\mathbf{L}^{2}}^{1-\frac{d}{2(m-1)}}\left\|\mathrm{D}^{m} U\right\|_{\mathbf{L}^{2}}^{\frac{d}{2(m-1)}} .
$$

In the same way as before, we obtain

$$
\|\mathrm{D} U\|_{\mathbf{L}^{\infty}} \leqslant C(1+t)^{s} Z,
$$

where

$$
\begin{aligned}
s & =-g_{1}\left(1-\frac{d}{2(m-1)}\right)-\left(g_{1}+m-1\right) \frac{d}{2(m-1)} \\
& =-g_{1}-\frac{d}{2}=\beta .
\end{aligned}
$$

3. Applying Lemma 4.4 to $\mathrm{D}^{2} U$ with $p=m-2$, which is possible since $m-2>d / 2$, we finally prove the third inequality.

Lemma 4.6. Let $f, \varphi \in\left(\mathbf{L}^{\infty} \cap \mathbf{H}^{m}\right)\left(\mathbb{R}^{d}\right)$, and let $\alpha \in \mathbb{N}^{d}$ such that $|\alpha|=k \leqslant m$. Then

$$
\left\|\partial^{\alpha}(f \varphi)\right\|_{\mathbf{L}^{2}} \leqslant C\left(\|f\|_{\mathbf{L}^{\infty}}\left\|\mathrm{D}^{k} \varphi\right\|_{\mathbf{L}^{2}}+\|\varphi\|_{\mathbf{L}^{\infty}}\left\|\mathrm{D}^{k} f\right\|_{\mathbf{L}^{2}}\right) .
$$

Lemma 4.7. Let $f \in\left(\mathbf{L}^{\infty} \cap \mathbf{H}^{m}\right)\left(\mathbb{R}^{d}\right)$. If $\nu \in \mathbb{N}$ and $\nu \geqslant 2$, or $\nu \in \mathbb{R}$ and $\nu \in[m,+\infty[$, we have $f^{\nu} \in \mathbf{H}^{m}\left(\mathbb{R}^{d}\right)$ and, for all $\alpha \in \mathbb{N}^{d}$ such that $|\alpha|=k \leqslant m$, we have

$$
\left\|\partial^{\alpha} f^{\nu}\right\|_{\mathbf{L}^{2}} \leqslant C\|f\|_{\mathbf{L}^{\infty}}^{\nu-1}\left\|\mathrm{D}^{k} f\right\|_{\mathbf{L}^{2}},
$$

where $C>0$ is a constant independent of $f, \alpha, \nu$.

Proof. For $\nu \in \mathbb{N}, \nu \geqslant 2$, we proceed by iteration on $\nu$, using Lemma 4.6.

For $\nu \in[m,+\infty[$, we have

$$
\partial^{\alpha}\left(f^{\nu}(x)\right)=\sum_{1 \leqslant j \leqslant k} \sum_{\substack{\beta_{1}+\ldots+\beta_{j}=\alpha \\\left|\beta_{i}\right|=b_{i} \geqslant 1}} c_{\alpha, \beta} f^{\nu-j} \partial^{\beta_{1}} f \ldots \partial^{\beta_{j}} f .
$$

Then we take $\partial^{\beta_{i}} f \in \mathbf{L}^{2 \frac{k}{b_{i}}}$, we apply Hölder inequality and Lemma 4.1 to obtain

$$
\begin{aligned}
\left\|\partial^{\alpha}\left(f^{\nu}\right)\right\|_{\mathbf{L}^{2}} & \leqslant C \sum_{j=1}^{k}\left\|f^{\nu-j}\right\|_{\mathbf{L}^{\infty}} \prod_{\substack{i=1 \\
\sum_{i=k}^{b_{i}=k}}}^{j}\left\|\partial_{\beta_{i}}^{b_{i}} f\right\|_{\mathbf{L}^{2} \frac{k}{b_{i}}} \\
& \leqslant C \sum_{j=1}^{k}\left\|f^{\nu-j}\right\|_{\mathbf{L}^{\infty}} \prod_{\substack{i=1 \\
\sum_{b_{i}=k}}}^{j}\|f\|_{\mathbf{L}^{\infty}}^{1-b_{i} / k}\left\|\mathrm{D}^{k} f\right\|_{\mathbf{L}^{2}}^{b_{i} / k} \\
& \leqslant C\|f\|_{\mathbf{L}^{\infty}}^{\nu-1}\left\|\mathrm{D}^{k} f\right\|_{\mathbf{L}^{2}},
\end{aligned}
$$

using in addition that, for all $j \in \llbracket 1, m \rrbracket,\left\|f^{\nu-j}\right\|_{\mathbf{L}^{\infty}} \leqslant\|f\|_{\mathbf{L}^{\infty}}^{\nu-j}$ since $\nu-j \geqslant 0$. 
Acknowledgment The author thanks Sylvie Benzoni-Gavage for initiating this work and for providing useful advice.

\section{References}

[1] J. R. Blake, editor. Acoustic cavitation and sonoluminescence. Royal Society, London, 1999. R. Soc. Lond. Philos. Trans. Ser. A Math. Phys. Eng. Sci. 357 (1999), no. 1751.

[2] J.-Y. Chemin. Dynamique des gaz à masse totale finie. Asymptotic Anal., 3(3):215-220, 1990.

[3] J.-Y. Chemin. Remarques sur l'apparition de singularités dans les écoulements eulériens compressibles. Comm. Math. Phys., 133(2):323-329, 1990.

[4] C. M. Dafermos. Hyperbolic conservation laws in continuum physics, volume 325 of Grundlehren der Mathematischen Wissenschaften. Springer-Verlag, Berlin, second edition, 2005.

[5] S. R. de Groot and P. Mazur. Nonequilibrium thermodynamics. Dover Publications Inc., New York, 1984. Reprint of the 1962 original.

[6] A. K. Evans. Instability of converging shock waves and sonoluminescence. Physical Review E, 54(5):5004-5011, 1996.

[7] M. Grassin. Global smooth solutions to Euler equations for a perfect gas. Indiana Univ. Math. J., 47(4):1397-1432, 1998.

[8] W. Gretler and R. Regenfelder. Variable-energy blast waves generated by a piston moving in a dusty gas. J. Engrg. Math., 52(4):321-336, 2005.

[9] J. Jena and V. D. Sharma. Self-similar shocks in a dusty gas. Internat. J. Non-Linear Mech., 34(2):313327, 1999.

[10] S. N. Kružkov. First order quasilinear equations with several independent variables. Mat. Sb. (N.S.), 81 (123):228-255, 1970.

[11] W. Lauterborn, T. Kurz, R. Geisler, D. Schanz, and O. Lindau. Acoustic cavitation, bubble dynamics and sonoluminescence. Ultrasonics sonochemistry, 14(4):484-491, 2007.

[12] T. T. Li. Global classical solutions for quasilinear hyperbolic systems, volume 32 of RAM: Research in Applied Mathematics. Masson, Paris, 1994.

[13] T. Makino, S. Ukai, and S. Kawashima. Sur la solution à support compact de l'équations d'Euler compressible. Japan J. Appl. Math., 3(2):249-257, 1986.

[14] R. Menikoff and B. J. Plohr. The Riemann problem for fluid flow of real materials. Rev. Modern Phys., 61(1):75-130, 1989.

[15] R. Racke. Lectures on nonlinear evolution equations. Aspects of Mathematics, E19. Friedr. Vieweg \& Sohn, Braunschweig, 1992. Initial value problems.

[16] P. G. Saffman. On the stability of laminar flow of a dusty gas. J. Fluid Mech., 13:120-128, 1962.

[17] D. Serre. Systèmes de lois de conservation. I. Fondations. Diderot Editeur, Paris, 1996. Hyperbolicité, entropies, ondes de choc.

[18] D. Serre. Systèmes de lois de conservation. II. Fondations. Diderot Editeur, Paris, 1996. Structures géométriques, oscillation et problèmes mixtes.

[19] D. Serre. Solutions classiques globales des équations d'Euler pour un fluide parfait compressible. Ann. Inst. Fourier (Grenoble), 47(1):139-153, 1997.

[20] T. C. Sideris. Formation of singularities in three-dimensional compressible fluids. Comm. Math. Phys., 101(4):475-485, 1985.

[21] T. C. Sideris. Delayed singularity formation in 2D compressible flow. Amer. J. Math., 119(2):371-422, 1997.

[22] H. Steiner and T. Hirschler. A self-similar solution of a shock propagation in a dusty gas. Eur. J. Mech. B Fluids, 21(3):371-380, 2002.

[23] M. E. Taylor. Partial differential equations. III, volume 117 of Applied Mathematical Sciences. SpringerVerlag, New York, 1997. Nonlinear equations, Corrected reprint of the 1996 original.

[24] J. P. Vishwakarma and G. Nath. A self- similar solution of a shock propagation in a mixture of a non-ideal gas and small solid particles. 44(4):239-254, 2009. 